\title{
PATENT PROTECTION \\ OF INTEGRATIVE TECHNICAL SYSTEM \\ - containing subsystems interconnected by elements of artificial intelligence and artificial neural networks. The use of analytical and algorithmic tools in the preparation of applications for the issuance of patent documents in the United States
}

\author{
Yerzhan Kusparmakov \\ Corresponding member of International Informatization Academy, Professional \\ Member of the New York Academy of Sciences, Master of Business \\ Administration, Head of Product management at SAMSUNG Electronics \\ e-mail:kusparmakov@gmail.com
}

\begin{abstract}
The integration of software solutions and mobile applications into innovative technical systems of all levels in the current process of transforming classical technologies into smart technologies and transforming the entire hierarchy of technical systems and their interconnections into smart technical systems is the basis for the formation of such innovative products that are most in demand on the markets.

As the practice of such innovative development shows, the use of methods and techniques of the inventive process adapted to today's elemental base and modern composite materials from generating an idea to the complete formation of materials for an application for an invention can be successfully carried out with the well-known Theory and Algorithm for solving inventive problems.

Of particular importance in the innovation process are relations with investors and potential strategic partners in the commercial application of the created innovative product.
\end{abstract}

Keywords: Patent, Licensing, Artificial intelligence, AI, Neural networks, Smart technology, Business, USA

Интеграция программных решений и мобильных приложений в инновационные технические системы всех уровней в сегодняшнем процессе превращения классических технологий в умные технологии и трансформации всей иерархии технических систем и их взаимосвязей в умные технические системы, является основой для формирования именно таких наиболее востребованных на рынках инновационных продуктов.

Как показывает практика такого инновационного развития, использование адаптированных под сегодняшнюю элементную базу и современные композитные материалы методов и приёмов изобретательского процесса от генерирования идеи до полного формирования материалов заявки на изобретение могут успешно вестись с известными Теорией и Алгоритмом решения изобретательских задач.

Применение ТРИЗ и АРИЗ конечно является только одним из многих возможных аналитических инструментов при составлении заявки на выдачу патента. 
Особое значение в инновационном процессе имеют отношения с инвесторами и потенциальными стратегическими партнёрами в деле коммерческого применения создаваемого инновационного продукта.

Для того, чтобы убедить инвестора в том, что вложения в предлагаемый проект принесут ему финансовый успех, необходимо детально изложить его экспертам суть проекта и раскрыть все технологические и инновационные секреты.

Сделать это невозможно без серьёзной защиты технических решений, положенных в основу инновационного продукта на всех этапах развития проекта.

Сейчас появились и успешно развиваются нетрадиционные виды и формы коллективного инвестирования и это также заставляет авторов проектов постоянно искать всё более и более надёжные формы защиты своих разработок.

Предварительная патентная заявка - Provisional Patent Applications, является и недорогим и надёжным вариантом защиты на период до 1 года.

Применение ТРИЗ и АРИЗ, как уже было сказано выше, при необходимом и достаточном обновлении основных приёмов и методов, является только одним из многих возможных инструментов при составлении заявки на выдачу патента и во многих случаях требует системной модификации и оптимизации с учётом новых инновационных обстоятельств.

Компанией заявителем или индивидуальным изобретателем, для каждого конкретного случая, с учётом всех обстоятельств, причин, целей и условий, с учётом результатов патентного прогнозирования, в полном соответствии с действующим патентным законодательством США (America Invents Act), может быть, а чаще всего необходима интегративная программа, в которой предельно точно разработана оригинальная система и структура заявки на выдачу патента.

Патентно-лицензионная стратегия, частью которой является последовательный процесс патентного прогнозирования, также может быть, а чаще всего - должна быть разработана для каждого конкретного заявителя или изобретателя.

Настоящая инструкция также полностью подходит и для составления заявочных материалов на подачу (Non Provisional Patent Application..., - Utility Patent Application). 


\section{APPARATUS AND METHOD FOR ...}

Название предполагаемого изобретения должно содержать не только краткое определение характера технического решения, но и предельно краткую коммерческую характеристику его влияния на рынок в пределах области использования предлагаемого технического решения. Название не должно содержать никаких рекламных характеристик..., - типа эффективный, наилучший и Т.П.

Очень важно в названии изобретения учесть коньюнктуру рынка, существующую на момент подачи заявки. В случае, если в цели авторов и (или их компаний и финансовых партнёров) и заявителей входит в дальнейшем привлечение дополнительных инвестиций в развитие проекта, базой которого должно явиться заявляемое техническое решение, желательно учесть в названии известные технологические и коммерческие интересы потенциальных партнёров.

Так как для патентного эксперта в США очень существенное значение имеет доказательство неочевидности заявляемого технического решения, то предельно важно учесть критерии и характеризацию неочевидности в названии изобретения.

Технические системы, относящиеся к так называемым умным технологиям и оборудованию, как правило должны включать программную часть, которая сама по себе техническим решением не является и не может классифицироваться как техническая система.

Но так как программные решения являются основой систем управления и контроля любой современной умной технологии, то и в названии изобретения это должно быть учтено, то есть название изобретения должно быть представлено в виде: Устройство, программа, система и метод; или - Аппарат, программа, система и метод.

Кроме того, при классификации технической системы, как умной системы, имеющей элементы искусственного интеллекта и искусственных нейронных сетей, необходимо иметь также накопители информации с соответствующим объёмом информации и соответствующим быстродействием.

Этот фактор должен быть отражён в названии, например..., - Аппарат, программа, система с накопителями памяти, и ассоциированный метод пользования. 


\section{REFERENCE TO RELATED APPLICATIONS}

[0001] This application claims the priority benefit under 35 U.S.C. §119(e) of U.S. Provisional Application Nos. 61/, filed...; and 61/, filed... The entire disclosures of each of these applications are incorporated herein by reference..., - в этом разделе публикуется информация о всех предварительных патентных заявках, которые были поданы авторами изобретения или заявителем до момента оформления и подачи настоящей заявки.

В случае, если предварительные патентные заявки в США (Provisional Patent Applications ) не подавались, то в этом разделе заявки приводится информация о всех предыдущих заявках по этой тематике и этому технологическому направлению, которые были поданы авторами изобретения или заявителем (от авторов настоящей заявки или от имени других авторов) до момента оформления и подачи настоящей заявки.

\section{TECHNICAL FIELD}

[0002] The invention relates to apparatus and methods for..., - в этом разделе приводят описание области техники и технологии к которым относится заявляемое техническое решение. Характер принадлежности к той или другой областям технологии должен быть предельно понятно аргументирован и связан с отличительными признаками заявляемого технического решения причинноследственными связями, охарактеризованными и сформулированными понятными для средней квалификации специалиста в этой области. Если отличительные признаки заявляемого технического решения являются очевидными для одной области и неочевидными для другой, то необходимо остановиться на этом и постараться дать аналитическое объяснение этому явлению.

Особенно важно охарактеризовать проблемы и противоречия характерные и очевидные для выбранного для изобретения технологического поля и в развитие этого тезиса для области техники и технологии, к которым относится заявляемое техническое решение.

В связи с тем, что в отличие от Европейского патентного агентства, в США при выполнении определённых условий, патент может быть выдан и на компьютерную программу, если это возможно, желательно иметь интеграционное решение по заявляемому техническому решению по типу..., - Программа, система, метод и устройство для их применения. 


\section{DESCRIPTION OF THE RELATED TECHNOLOGY}

[0003] - этот раздел состоит из двух частей. В первой части даётся более специфицированная, конкретная, локальная аналитическая оценка области техники и технологии, к которой относится заявляемое техническое решение. Даётся описание наиболее характерных особенностей технологии, её известных недостатков и преимуществ.

[0004] - в этой части раздела желательно начать поиск и предварительный анализ противоречий общего характера, которые являются общеизвестными и характерными для технологии к которым авторы изобретения его относят.

\section{BACKGROUND OF THE INVENTION}

[0005] - в этом разделе, который является одним из самых важных в заявке, даётся глубокий анализ результатов патентного поиска по всем вариантам и источникам опубликованной патентной информации, как минимум по Патентному ведомству США, лучше, если и по Европейскому патентному ведомству, и ещё лучше если привести сравнительный анализ известных технических решений и в завершение привести результаты структурного поэлементного анализа.

Необходимо после этого, используя инструменты ТРИЗ и АРИЗ провести поиск, формулировку и сравнительный анализ всех видов противоречий в известных технических решениях и определить и охарактеризовать возможный идеальный конечный результат.

После этого проанализировать возможность в заявляемом техническом решении принципиального достижения идеального конечного результата при использовании техники, технологии, материалов и средств контроля и управления, известных по состоянию на момент подачи заявки.

\section{SUMMARY}

[0006] According to some embodiments of the invention..., - в этом разделе даётся определение первой локальной конечной цели изобретения и даётся определение пути её достижения в рамках соответствия пунктам формулы изобретения; приводится идентификация поставленной локальной цели и определяется достаточно ли достичь поставленную цель, что бы констатировать достижение по крайней мере частичного или локального идеального конечного результата. 
[0007] In some embodiments, programs, systems and methods of..., - в этом разделе даётся определение второй локальной конечной цели изобретения и даётся определение пути её достижения в рамках соответствия пунктам формулы изобретения; приводится идентификация поставленной локальной цели и определяется достаточно ли достичь поставленную цель, что бы констатировать достижение по крайней мере частичного или локального идеального конечного результата.

[0008] In some embodiments, further programs, systems and methods for..., - в этом разделе даётся определение третьей локальной конечной цели изобретения и даётся определение пути её достижения в рамках соответствия пунктам формулы изобретения; приводится идентификация поставленной локальной цели и определяется достаточно ли достичь поставленную цель, что бы констатировать достижение по крайней мере частичного или локального идеального конечного результата.

[0009] Also in some further embodiments, programs, systems and methods of..., - в этом разделе даётся определение первой интегральной локальной конечной цели изобретения и даётся определение пути её достижения в рамках соответствия пунктам формулы изобретения; приводится идентификация поставленной интегральной локальной цели и определяется достаточно ли достичь поставленную цель, что бы констатировать достижение по крайней мере частичного или локального идеального конечного результата.

[0010] In some embodiments, an apparatus is provided with..., - в этом разделе даётся определение первой локальной конечной цели изобретения, связанной причинноследственной связью со второй локальной конечной целью изобретения и даётся определение пути достижения указанной целевой интеграции в рамках соответствия пунктам формулы изобретения; приводится идентификация поставленной локальной интегрированной цели и определяется достаточно ли достичь эту поставленную цель, что бы констатировать достижение по крайней мере частичного или локального идеального конечного результата.

[0011] - в этом разделе даётся определение первой локальной конечной цели изобретения, связанной причинно-следственной связью со второй и третьей локальной конечной целью изобретения и даётся определение пути достижения указанной целевой интеграции в рамках соответствия пунктам формулы изобретения; приводится идентификация поставленной локальной интегрированной цели и определяется достаточно ли достичь эту поставленную цель, что бы 
констатировать достижение по крайней мере частичного или локального идеального конечного результата.

[0012] In some embodiments, an apparatus for..., - в этом разделе даётся определение первой локальной конечной цели изобретения в части решений по аппарату, связанной причинно-следственной связью со второй локальной конечной целью изобретения, также отнесённой к аппарату и даётся определение пути достижения указанной целевой интеграции в рамках соответствия пунктам формулы изобретения ; приводится идентификация поставленной локальной интегрированной цели и определяется достаточно ли достичь эту поставленную цель, что бы констатировать достижение по крайней мере частичного или локального идеального конечного результата.

[0013] In some other embodiments, an apparatus is provided with a system for...,- в этом разделе к аппарату прибавляют одну из известных систем, которая необходима для его декларированного рабочего цикла и анализируют наличие или отсутствие скачка эффекта.

[0014] In some other embodiments, an apparatus is provided with a system for...,- в этом разделе к аппарату прибавляют вторую из известных систем, которая необходима для его декларированного рабочего цикла и анализируют наличие или отсутствие скачка эффекта.

[0015] In some embodiments of the various apparatus, the...,- в этом разделе к аппарату прибавляют сочетание из известных систем, интегрированных с аппаратом в интегративную систему, которая необходима для его декларированного рабочего цикла и анализируют наличие или отсутствие скачка эффекта.

[0016] - в этом разделе к аппарату прибавляют одну за одной комбинацию из известных систем, которая необходима для его декларированного рабочего цикла и анализируют наличие или отсутствие скачка эффекта.

[0017] The system for..., - в этом разделе проводят структурный анализ системы (систем) технического решения и отслеживают достаточность наличия в техническом решении сочетания всех необходимых систем для обеспечения достижения идеального конечного результата.

[0018] Structurally, the apparatus can be provided with а..., - в этом разделе проводят структурный анализ узлов и компонентов аппарата. 
[0019] In the apparatus, the system for..., - в этом разделе показывают, как влияют конструктивные отличительные признаки на достижение идеального конечного результата.

[0020] The apparatus can also comprise a system for...,- в этом разделе рассматривают возможность включения в аппарат различных дополнительных функционально независимых и автономных систем, которые могут усилить эффект от использования предлагаемого изобретения и тем самым обеспечить более уверенное достижение идеального конечного результата.

[0021] In some embodiments, the method of operation of the device involves..., - в этом разделе анализируют локальные и интегральные цели в динамике или действии, анализируют методы достижения целей и сравнивают параметры после достижения указанной цели с идеальным конечным результатом.

[0022] An external...,- в этом разделе рассматриваются дополнительные возможные аспекты усовершенствования и модификации целей и способов их достижения и анализируется, и оценивается потенциал достижения дополнительных компонентов идеального конечного результата.

[0023] The following can be provided to..., - в этом разделе расцениваются различные действия и конструктивные усовершенствования способные дополнить сущность идеального конечного результата новым содержанием.

[0024] In some embodiments..., - в этом разделе даётся окончательное определение полностью интегрированной локальной конечной цели изобретения и даётся определение пути её достижения в рамках соответствия пунктам формулы изобретения ; приводится идентификация поставленной локальной, полностью интегрированной цели и определяется достаточно ли достичь поставленную цель, что бы констатировать достижение по крайней мере частичного или локального идеального конечного результата.

\section{BRIEF DESCRIPTION OF THE DRAWINGS}

[0025] The invention will be better understood from the Detailed Description and from the appended drawings, which are meant to illustrate and not to limit the invention. The Figures are not necessarily drawn to scale, nor are the relative sizes of parts within the Figures necessary in proportional to one another, - изобретение будет лучше понято из подробного описания и прилагаемых чертежей, которые предназначены для иллюстрации, а не для ограничения изобретения. Фигуры не обязательно должны 
быть изображены в масштабе, и относительные размеры частей внутри фигуры не должны быть пропорциональны друг другу.

[0026] Figure 1 is an example of a..., - в этой фигуре представляют общую принципиальную блок-схему технической системы/надсистемы, в которой представлены все подсистемы и их связи между собой и выходы и линии связи с надсистемой с концентрацией внимания на функциональных особенностях и отличиях интегрированных в надсистему программных элементов в совокупности с системами управления и контроля надсистемы содержащих элементы искусственного интеллекта и искусственные нейронные сети.

[0027] Figure 2 is an example of a ..., - в этой фигуре представляют общую принципиальную блок-схему ведущей и центральной технической системы/подсистемы, в которой представлены все функционально связанные с ней подсистемы и их связи между собой и выходы и линии связи с надсистемой с концентрацией внимания на функциональных особенностях и отличиях интегрированных в надсистему и по возможности в подсистемы программных элементов в совокупности с системами управления и контроля надсистемы содержащих элементы искусственного интеллекта и искусственные нейронные сети.

Также отражаются технические детали и программные функции, и алгоритмы характеризующие неочевидность этих решений в сочетании с общими принципами применения элементов искусственного интеллекта и искусственных нейронных сетей.

[0028] Figure 3 shows an example..., - в этой фигуре представляют общую принципиальную блок-схему вспомогательной или локальной первой технической системы/подсистемы, в которой представлены все функционально связанные с ней подсистемы и их связи между собой и выходы и линии связи с надсистемой с концентрацией внимания на функциональных особенностях и отличиях интегрированных в надсистему и по возможности в подсистемы программных элементов в совокупности с системами управления и контроля надсистемы содержащих элементы искусственного интеллекта и искусственные нейронные сети.

Также отражаются технические детали и программные функции, и алгоритмы характеризующие неочевидность этих решений в сочетании с общими принципами применения элементов искусственного интеллекта и искусственных нейронных сетей.

[0029] Figure 4 shows an example of components of the..., - в этой фигуре представляют общую принципиальную блок-схему вспомогательной или 
локальной второй технической системы/подсистемы, в которой представлены все функционально связанные с ней подсистемы и их связи между собой и выходы и линии связи с надсистемой с концентрацией внимания на функциональных особенностях и отличиях интегрированных в надсистему и по возможности в подсистемы программных элементов в совокупности с системами управления и контроля надсистемы содержащих элементы искусственного интеллекта и искусственные нейронные сети.

Также отражаются технические детали и программные функции, и алгоритмы характеризующие неочевидность этих решений в сочетании с общими принципами применения элементов искусственного интеллекта и искусственных нейронных сетей.

[0030] Figure 5 shows an example of a..,, - в этой фигуре представляют общую принципиальную блок-схему вспомогательной или локальной третьей технической системы/подсистемы, в которой представлены все функционально связанные с ней подсистемы и их связи между собой и выходы и линии связи с надсистемой с концентрацией внимания на функциональных особенностях и отличиях интегрированных в надсистему и по возможности в подсистемы программных элементов в совокупности с системами управления и контроля надсистемы содержащих элементы искусственного интеллекта и искусственные нейронные сети.

Также отражаются технические детали и программные функции, и алгоритмы характеризующие неочевидность этих решений в сочетании с общими принципами применения элементов искусственного интеллекта и искусственных нейронных сетей.

[0031] Figure 6 is a..., - иллюстрации к фигуре 1, выполненные как зависимые графические структуры к зависимым пунктам формулы изобретения, относящимся к независимому пункту 1.

[0032] Figure 7 shows an example of a...,- иллюстрации к фигуре 2, выполненные как зависимые графические структуры к зависимым пунктам формулы изобретения, относящимся к независимому пункту 2.

[0033] Figure 8 is an example of a..., - иллюстрации к фигуре 3, выполненные как зависимые графические структуры к зависимым пунктам формулы изобретения, относящимся к независимому пункту 3.

[0034] Figure 9 is an example of a..., - иллюстрации к фигуре 4, выполненные как зависимые графические структуры к зависимым пунктам формулы изобретения, относящимся к независимому пункту 4. 
[0035] Figure 10 is an example of a...,- иллюстрации к фигуре 5, выполненные как зависимые графические структуры к зависимым пунктам формулы изобретения, относящимся к независимому пункту 5.

[0036] Figure 11 is an example of a..., - в этой фигуре представляют общую принципиальную блок-схему или алгоритм взаимодействия вспомогательной или локальной первой технической системы/подсистемы, в которой представлены все функционально связанные с ней подсистемы и их связи между собой и выходы и линии связи с надсистемой с концентрацией внимания на функциональных особенностях и отличиях интегрированных в надсистему и по возможности в подсистемы программных элементов в совокупности с системами управления и контроля надсистемы содержащих элементы искусственного интеллекта и искусственные нейронные сети.

Также отражаются технические детали и программные функции, и алгоритмы характеризующие неочевидность этих решений в сочетании с общими принципами применения элементов искусственного интеллекта и искусственных нейронных сетей.

[0037] Figure 12 is an example of an..., - в этой фигуре представляют общую принципиальную блок-схему или алгоритм взаимодействия вспомогательной или локальной второй по значимости технической системы/подсистемы, в которой представлены все функционально связанные с ней подсистемы и их связи между собой и выходы и линии связи с надсистемой с концентрацией внимания на функциональных особенностях и отличиях интегрированных в надсистему и по возможности в подсистемы программных элементов в совокупности с системами управления и контроля надсистемы содержащих элементы искусственного интеллекта и искусственные нейронные сети.

Также отражаются технические детали и программные функции, и алгоритмы характеризующие неочевидность этих решений в сочетании с общими принципами применения элементов искусственного интеллекта и искусственных нейронных сетей.

[0038] Figure 13 is an example of a..., - в этой фигуре представляют общую принципиальную блок-схему или алгоритм взаимодействия вспомогательной или локальной третьей по значимости технической системы/подсистемы, в которой представлены все функционально связанные с ней подсистемы и их связи между собой и выходы и линии связи с надсистемой с концентрацией внимания на функциональных особенностях и отличиях интегрированных в надсистему и по возможности в подсистемы программных элементов в совокупности с системами 
управления и контроля надсистемы содержащих элементы искусственного интеллекта и искусственные нейронные сети.

Также отражаются технические детали и программные функции, и алгоритмы характеризующие неочевидность этих решений в сочетании с общими принципами применения элементов искусственного интеллекта и искусственных нейронных сетей

[0039] Figure 14 is an example of a..., - в этой фигуре представляют общую принципиальную блок-схему или алгоритм взаимодействия вспомогательной или локальной четвёртой по значимости технической системы/подсистемы, в которой представлены все функционально связанные с ней подсистемы и их связи между собой и выходы и линии связи с надсистемой с концентрацией внимания на функциональных особенностях и отличиях интегрированных в надсистему и по возможности в подсистемы программных элементов в совокупности с системами управления и контроля надсистемы содержащих элементы искусственного интеллекта и искусственные нейронные сети.

Также отражаются технические детали и программные функции, и алгоритмы характеризующие неочевидность этих решений в сочетании с общими принципами применения элементов искусственного интеллекта и искусственных нейронных сетей

[0040] Figure 15 is an example of a..., - в этой фигуре представляют общую принципиальную блок-схему или алгоритм взаимодействия вспомогательной или локальной пятой по значимости технической системы/подсистемы, в которой представлены все функционально связанные с ней подсистемы и их связи между собой и выходы и линии связи с надсистемой с концентрацией внимания на функциональных особенностях и отличиях интегрированных в надсистему и по возможности в подсистемы программных элементов в совокупности с системами управления и контроля надсистемы содержащих элементы искусственного интеллекта и искусственные нейронные сети.

Также отражаются технические детали и программные функции, и алгоритмы характеризующие неочевидность этих решений в сочетании с общими принципами применения элементов искусственного интеллекта и искусственных нейронных сетей.

[0041] Figure 16 is an example of a..., - иллюстрации к фигуре 11, выполненные как зависимые графические структуры к зависимым пунктам формулы изобретения, относящимся к независимому пункту 11. 
[0042] Figure 17 shows an example of..., - иллюстрации к фигуре 12, выполненные как зависимые графические структуры к зависимым пунктам формулы изобретения, относящимся к независимому пункту 12.

[0043] Figure 18 is an example of a...,- иллюстрации к фигуре 13, выполненные как зависимые графические структуры к зависимым пунктам формулы изобретения, относящимся к независимому пункту 13.

[0044] Figure 19 is an example of a.....,., - иллюстрации к фигуре 14, выполненные как зависимые графические структуры к зависимым пунктам формулы изобретения, относящимся к независимому пункту 14.

[0045] Figure 20 is an example of a..., - иллюстрации к фигуре 15, выполненные как зависимые графические структуры к зависимым пунктам формулы изобретения, относящимся к независимому пункту 15.

[0046] Figure 21 is an example of a..., - в этой фигуре представляют общую принципиальную блок-схему или алгоритм комплексной интеграции в центральную надсистему - вспомогательной или локальной первой по значимости технической системы/подсистемы, в которой представлены все функционально связанные с ней подсистемы и их связи между собой и выходы и линии связи с надсистемой с концентрацией внимания на функциональных особенностях и отличиях интегрированных в надсистему и по возможности в подсистемы программных элементов в совокупности с системами управления и контроля надсистемы содержащих элементы искусственного интеллекта и искусственные нейронные сети.

Также отражаются технические детали и программные функции, и алгоритмы характеризующие неочевидность этих решений в сочетании с общими принципами применения элементов искусственного интеллекта и искусственных нейронных сетей.

[0047] Figure 22 is an example of a..., - в этой фигуре представляют общую принципиальную блок-схему или алгоритм комплексной интеграции в центральную надсистему - вспомогательной или локальной второй по значимости технической системы/подсистемы, в которой представлены все функционально связанные с ней подсистемы и их связи между собой и выходы и линии связи с надсистемой с концентрацией внимания на функциональных особенностях и отличиях интегрированных в надсистему и по возможности в подсистемы программных элементов в совокупности с системами управления и контроля 
надсистемы содержащих элементы искусственного интеллекта и искусственные нейронные сети.

Также отражаются технические детали и программные функции, и алгоритмы характеризующие неочевидность этих решений в сочетании с общими принципами применения элементов искусственного интеллекта и искусственных нейронных сетей.

[0048] Figure 23 is an example of a..., - в этой фигуре представляют общую принципиальную блок-схему или алгоритм комплексной интеграции в центральную надсистему - вспомогательной или локальной третьей по значимости технической системы/подсистемы, в которой представлены все функционально связанные с ней подсистемы и их связи между собой и выходы и линии связи с надсистемой с концентрацией внимания на функциональных особенностях и отличиях интегрированных в надсистему и по возможности в подсистемы программных элементов в совокупности с системами управления и контроля надсистемы содержащих элементы искусственного интеллекта и искусственные нейронные сети.

Также отражаются технические детали и программные функции, и алгоритмы характеризующие неочевидность этих решений в сочетании с общими принципами применения элементов искусственного интеллекта и искусственных нейронных сетей.

[0049] Figure 24 is an example of a..., - в этой фигуре представляют общую принципиальную блок-схему или алгоритм комплексной интеграции в центральную надсистему - вспомогательной или локальной четвёртой по значимости технической системы - подсистемы, в которой представлены все функционально связанные с ней подсистемы и их связи между собой и выходы и линии связи с надсистемой с концентрацией внимания на функциональных особенностях и отличиях интегрированных в надсистему и по возможности в подсистемы программных элементов в совокупности с системами управления и контроля надсистемы содержащих элементы искусственного интеллекта и искусственные нейронные сети.

Также отражаются технические детали и программные функции, и алгоритмы характеризующие неочевидность этих решений в сочетании с общими принципами применения элементов искусственного интеллекта и искусственных нейронных сетей. 


\section{DETAILED DESCRIPTION}

[0050] According to some embodiments, an..., - в этом разделе показывают элементы существенной новизны, содержащиеся в предполагаемом изобретении на уровне основного технического решения верхнего положения в иерархии новых или обновлённых технических решений и их интегративных сочетаний.

[0051] In some embodiments, a..., - в этом разделе показывают принципы интеграции программных решений в основном техническом решении.

[0052] In order to develop..., - в этом разделе показывают принципиальные элементы развития всех видов технических решений на уровне основного технического решения без интеграции с программными и контрольными решениями.

[0053] To develop a..., - в этом разделе показывают методы и процессы, а также алгоритмы предназначенные для глубокой интеграции программных решений всех видов в комплексе и иерархии основного технического решения и его интегративных сочетаний с техническими подсистемами.

[0054] It will be appreciated that some..., - в этом разделе показывают увязку и следствие ввода программных решений в принципиальную характеристику и связи во всех взаимосвязанных и взаимно интегрированных технических системах с учётом насущных элементов искусственного интеллекта и связанных с ними искусственных нейронных сетей.

[0055] In some embodiments, the..., - в этом разделе приводят характеристики и новые отличительные признаки, появившиеся при интеграции программных решений всех уровней в основное техническое решение.

[0056] It will be appreciated that the..., - в этом разделе показывают элементы существенной новизны, возникшие при интеграции программных решений в классические по типу технические решения всех уровней иерархии.

[0057] In some embodiments, the..., - в этом разделе приводят развёрнутые характеристики и рабочие связи элементов искусственного интеллекта с основной схемой работы технических систем всех уровней иерархии с привязкой принципов существенной новизны к алгоритму или принципам действия всей комплексной изобретённой технической системы.

[0058] In some embodiments, an..., - в этом разделе приводят развёрнутые характеристики и рабочие связи элементов искусственного интеллекта и искусственных нейронных сетей с основной схемой работы технических систем 
всех уровней иерархии с привязкой принципов существенной новизны к алгоритму или принципам действия всей комплексной изобретённой технической системы.

[0059] Reference will now be made to the figures, in which like numerals refer to like parts throughout.

[0060] In Figure 1..., - в этом разделе дают развернутое описание Фигуры 1 с расшифровкой всех условных обозначений и номеров позиций или цифровых обозначений. Все цифровые обозначения для этой фигуры должны иметь вид - 101, $102 \ldots$ The following reference numerals identify the following features.

[0061] Figure 2 shows an example of a..., - в этом разделе дают развернутое описание Фигуры 2 с расшифровкой всех условных обозначений и номеров позиций или цифровых обозначений. Все цифровые обозначения для этой фигуры должны иметь вид $-201,202 \ldots$ The following reference numerals identify the following features.

[0062] Figure 3 shows a...,- в этом разделе дают развернутое описание Фигуры 3 с расшифровкой всех условных обозначений и номеров позиций или цифровых обозначений. Все цифровые обозначения для этой фигуры должны иметь вид - 301, $302 \ldots$ The following reference numerals identify the following features.

[0063] Figure 4 shows a..., - в этом разделе дают развернутое описание Фигуры 4 с расшифровкой всех условных обозначений и номеров позиций или цифровых обозначений. Все цифровые обозначения для этой фигуры должны иметь вид - 401, $402 \ldots$ The following reference numerals identify the following features.

[0064] Figure 5 shows a..., - в этом разделе дают развернутое описание Фигуры 5 с расшифровкой всех условных обозначений и номеров позиций или цифровых обозначений. Все цифровые обозначения для этой фигуры должны иметь вид - 501, $502 \ldots$ The following reference numerals identify the following features.

[0065] Figure 6 shows a..., - в этом разделе дают развернутое описание Фигуры 6 c расшифровкой всех условных обозначений и номеров позиций или цифровых обозначений. Все цифровые обозначения для этой фигуры должны иметь вид - 601 , $602 \ldots$ The following reference numerals identify the following features.

[0066] Figure 7 shows..., - в этом разделе дают развернутое описание Фигуры 7 с расшифровкой всех условных обозначений и номеров позиций или цифровых обозначений. Все цифровые обозначения для этой фигуры должны иметь вид - 701, $702 \ldots$ The following reference numerals identify the following features. 
[0067] Figure 8 shows an...,- в этом разделе дают развернутое описание Фигуры 8 c расшифровкой всех условных обозначений и номеров позиций или цифровых обозначений. Все цифровые обозначения для этой фигуры должны иметь вид -801 , $802 \ldots$ The following reference numerals identify the following features.

[0068] Figure 9 shows a..., - в этом разделе дают развернутое описание Фигуры 9 с расшифровкой всех условных обозначений и номеров позиций или цифровых обозначений. Все цифровые обозначения для этой фигуры должны иметь вид - 901, $902 \ldots$ The following reference numerals identify the following features.

[0069] Figure 10 shows a..., - в этом разделе дают развернутое описание Фигуры 10 с расшифровкой всех условных обозначений и номеров позиций или цифровых обозначений. Все цифровые обозначения для этой фигуры должны иметь вид - 1001, $1002 \ldots$ The following reference numerals identify the following features.

[0070] Figure 11 shows..., - в этом разделе дают развернутое описание Фигуры 11 с расшифровкой всех условных обозначений и номеров позиций или цифровых обозначений. Все цифровые обозначения для этой фигуры должны иметь вид - 1101, $1102 \ldots$ The following reference numerals identify the following features.

[0071] Figure12 shows ..., - в этом разделе дают развернутое описание Фигуры 12 с расшифровкой всех условных обозначений и номеров позиций или цифровых обозначений. Все цифровые обозначения для этой фигуры должны иметь вид, -1201, $1202 \ldots$ The following reference numerals identify the following features.

[0072] Figure 13 shows a...,- в этом разделе дают развернутое описание Фигуры 13 с расшифровкой всех условных обозначений и номеров позиций или цифровых обозначений. Все цифровые обозначения для этой фигуры должны иметь вид - 1301, $1302 \ldots$ The following reference numerals identify the following features.

[0073] Figure 14 shows..., - в этом разделе дают развернутое описание Фигуры 14 с расшифровкой всех условных обозначений и номеров позиций или цифровых обозначений. Все цифровые обозначения для этой фигуры должны иметь вид - 1401, $1402 \ldots$ The following reference numerals identify the following features.

[0074] Figure 15 shows a..., - в этом разделе дают развернутое описание Фигуры 15 с расшифровкой всех условных обозначений и номеров позиций или цифровых обозначений. Все цифровые обозначения для этой фигуры должны иметь вид - 1501, $1502 \ldots$ The following reference numerals identify the following features. 
[0075] Figure 16 shows a..., - в этом разделе дают развернутое описание Фигуры 16 с расшифровкой всех условных обозначений и номеров позиций или цифровых обозначений. Все цифровые обозначения для этой фигуры должны иметь вид - 1601 , $1602 \ldots$ The following reference numerals identify the following features.

[0076] Figure 17 shows..., - в этом разделе дают развернутое описание Фигуры 17 с расшифровкой всех условных обозначений и номеров позиций или цифровых обозначений. Все цифровые обозначения для этой фигуры должны иметь вид - 1701, $1702 \ldots$ The following reference numerals identify the following features.

[0077] Figure 18 shows a..., - в этом разделе дают развернутое описание Фигуры 18 с расшифровкой всех условных обозначений и номеров позиций или цифровых обозначений. Все цифровые обозначения для этой фигуры должны иметь вид - 1801 , $1802 \ldots$ The following reference numerals identify the following features.

[0078] Figure 19 shows a..., - в этом разделе дают развернутое описание Фигуры 19 с расшифровкой всех условных обозначений и номеров позиций или цифровых обозначений. Все цифровые обозначения для этой фигуры должны иметь вид - 1901, $1902 \ldots$ The following reference numerals identify the following features.

[0079] Figure 20 shows..., - в этом разделе дают развернутое описание Фигуры 20 с расшифровкой всех условных обозначений и номеров позиций или цифровых обозначений. Все цифровые обозначения для этой фигуры должны иметь вид - 2001, $2002 \ldots$ The following reference numerals identify the following features.

[0080] Figure 21 shows a..., - в этом разделе дают развернутое описание Фигуры 21 с расшифровкой всех условных обозначений и номеров позиций или цифровых обозначений. Все цифровые обозначения для этой фигуры должны иметь вид -2101 , $2102 \ldots$ The following reference numerals identify the following features.

[0081] Figure 22 shows an..., - в этом разделе дают развернутое описание Фигуры 22 с расшифровкой всех условных обозначений и номеров позиций или цифровых обозначений. Все цифровые обозначения для этой фигуры должны иметь вид -2201 , $2202 \ldots$ The following reference numerals identify the following features.

[0082] Figure 23 shows a...,- в этом разделе дают развернутое описание Фигуры 23 с расшифровкой всех условных обозначений и номеров позиций или цифровых обозначений. Все цифровые обозначения для этой фигуры должны иметь вид - 2301, $2302 \ldots$ The following reference numerals identify the following features. 
[0083] Figure 24 shows a..., - в этом разделе дают развернутое описание Фигуры 24 с расшифровкой всех условных обозначений и номеров позиций или цифровых обозначений. Все цифровые обозначения для этой фигуры должны иметь вид - 2401, $2402 \ldots$ The following reference numerals identify the following features.

\section{TEST RESULTS}

[0084] В этом разделе демонстрируются результаты испытаний в виде показателей, таблиц, графиков и диаграмм.

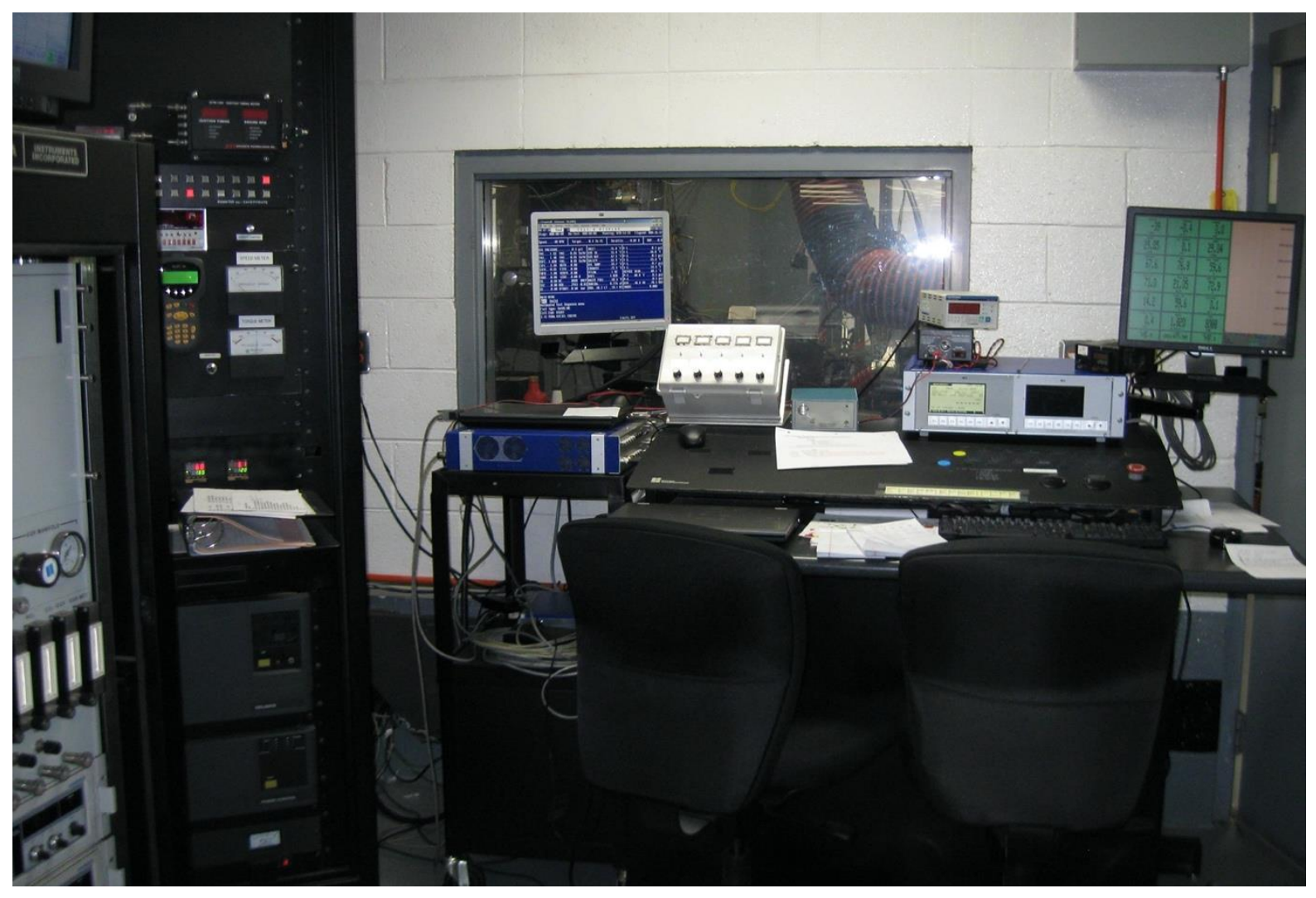

Рисунок 1, - на рисунке показан пример расположения системы аналитического и контрольно-измерительного оборудования испьтательного стенда, предназначенного для испытаний и аналитической оценки дизельного двигателя.

Системы стенда включают элементы искусственного интеллекта и искусственные нейронные сети. Очень важно показать и раскрыть новизну применения этой системы для достижения идеального конечного результата или его эквивалента применительно к заявляемому материалу 


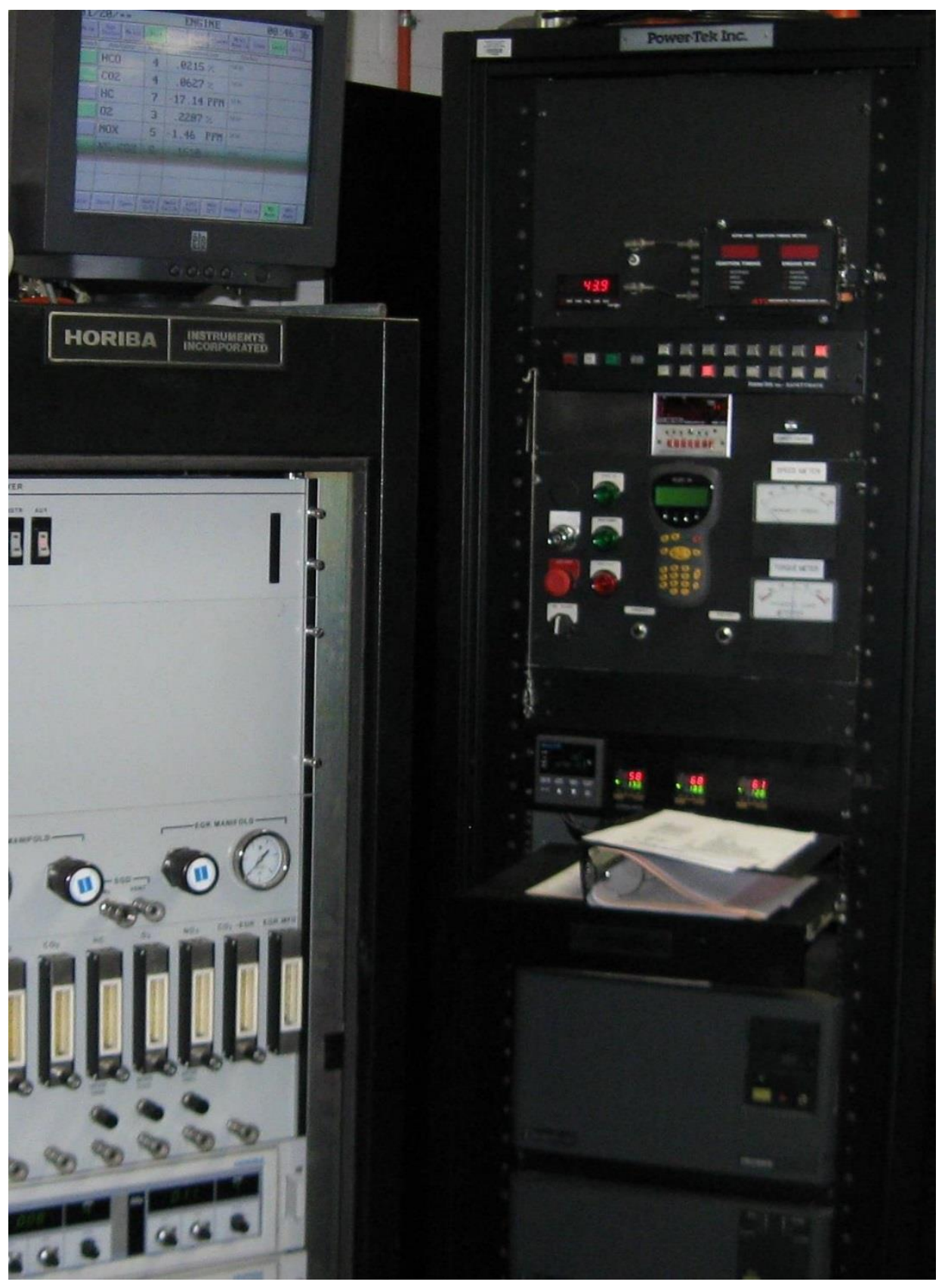

Рисунок 2, - на рисунке показан пример фрагмента расположения системь аналитического и контрольно-измерительного оборудования испьтательного стенда, предназначенного для испытаний и аналитической оценки дизельного двигателя. Системы стенда включают элементы искусственного интеллекта и искусственные нейронные сети. Очень важно показать и раскрыть новизну применения этой системы для достижения идеального конечного результата или его эквивалента применительно к заявляемому материалу, а также показать взаимодействие контрольно-аналитических компонентов системы с элементами искусственного интеллекта 


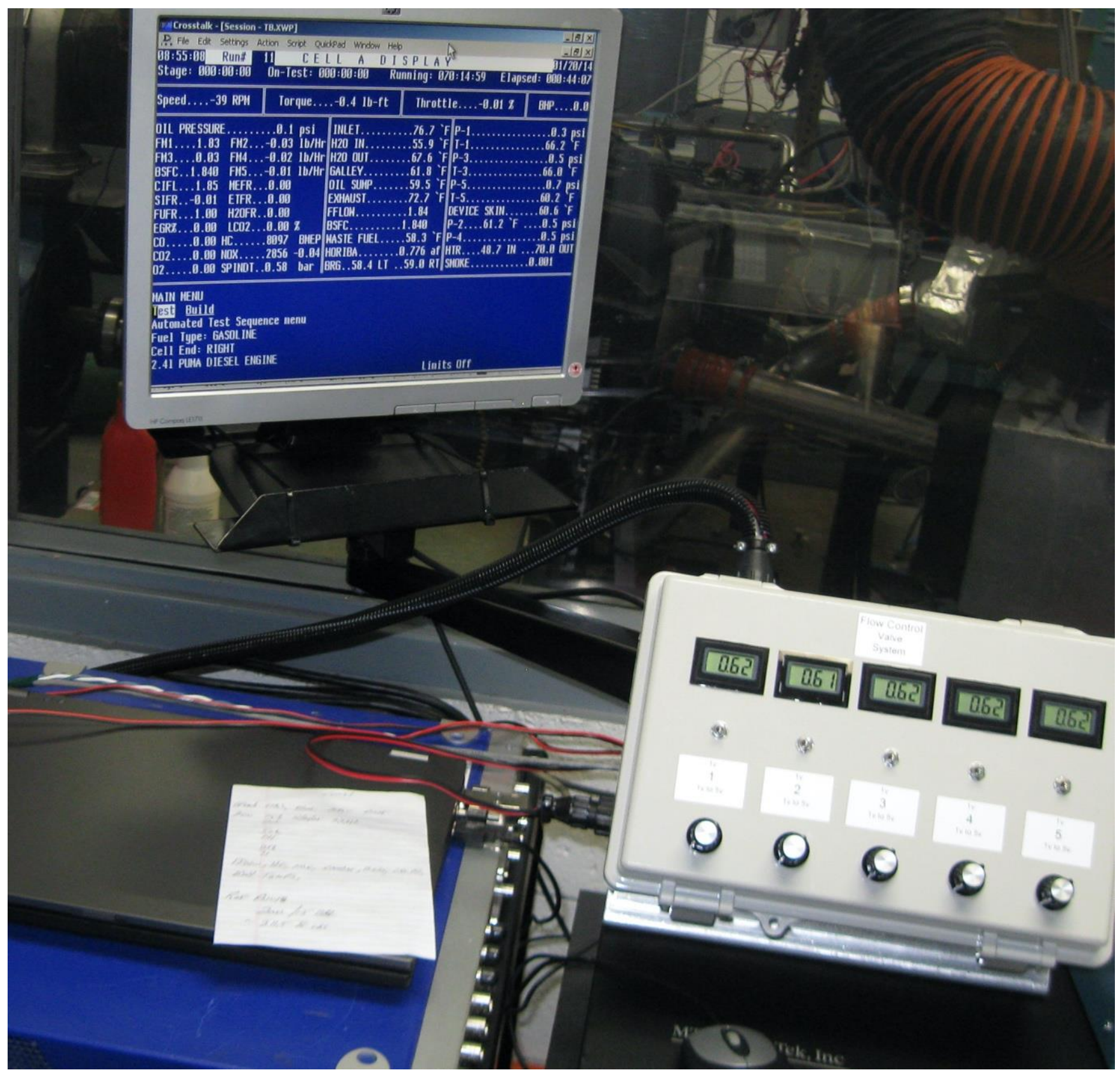

Рисунок 3, - на рисунке показан пример расположения системы аналитического и контрольно-измерительного оборудования испьтательного стенда, предназначенного для испытаний и аналитической оценки дизельного двигателя.

Системы стенда включают элементы искусственного интеллекта и искусственные нейронные сети. Очень важно в материалах заявки показать и раскрыть новизну применения этой системы для достижения идеального конечного результата или его эквивалента применительно к заявляемому материалу 


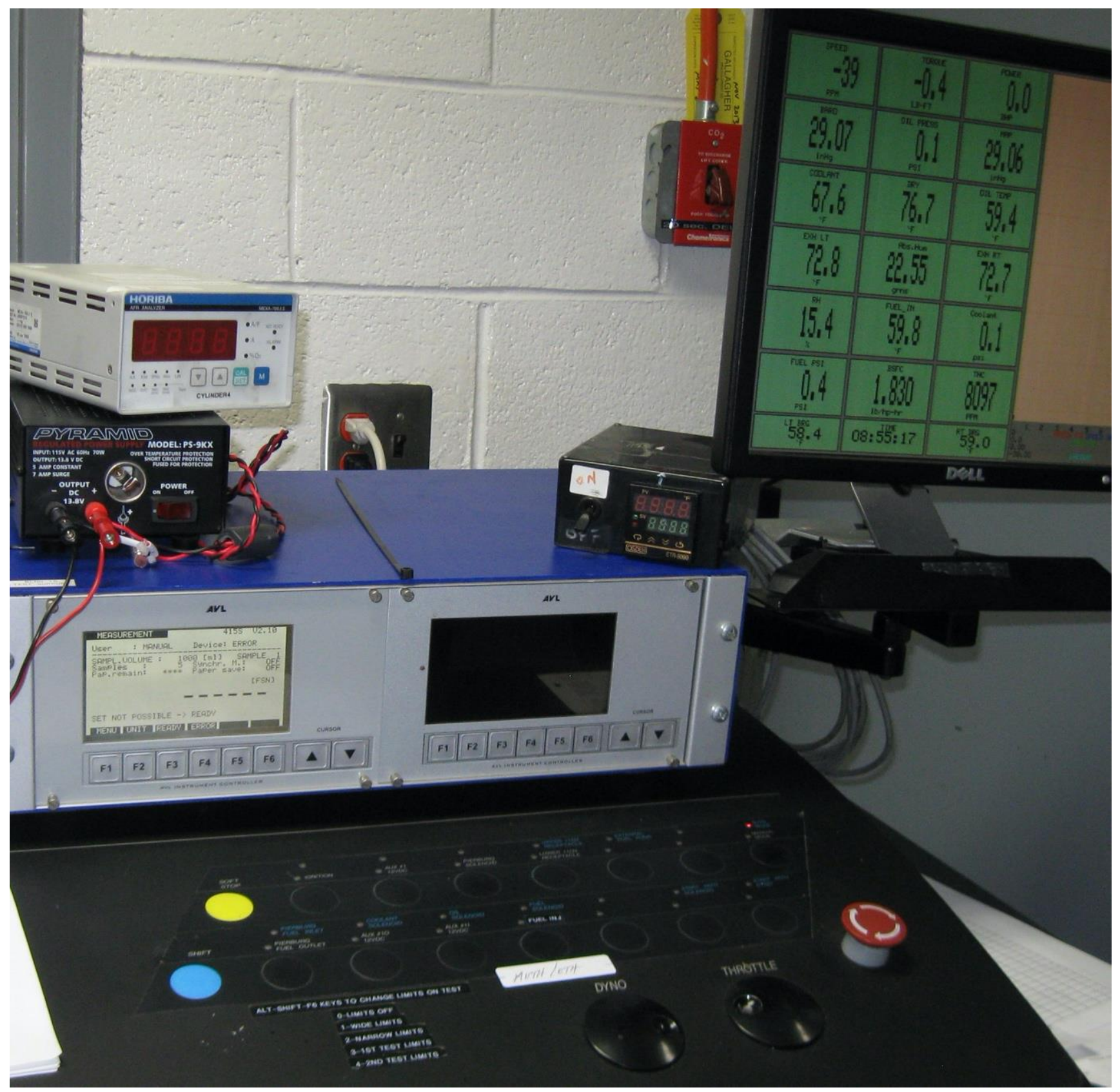

Рисунок 4, - на рисунке показан пример фрагмента расположения системы аналитического и контрольно-измерительного оборудования испьтательного стенда, предназначенного для испытаний и аналитической оченки дизельного двигателя. Системы стенда включают элементы искусственного интеллекта и искусственные нейронные сети. Очень важно показать и раскрыть новизну применения этой системы для достижения идеального конечного результата или его эквивалента применительно к заявляемому материалу, а также показать 
взаимодействие контрольно-аналитических компонентов системы с элементами искусственного интеллекта
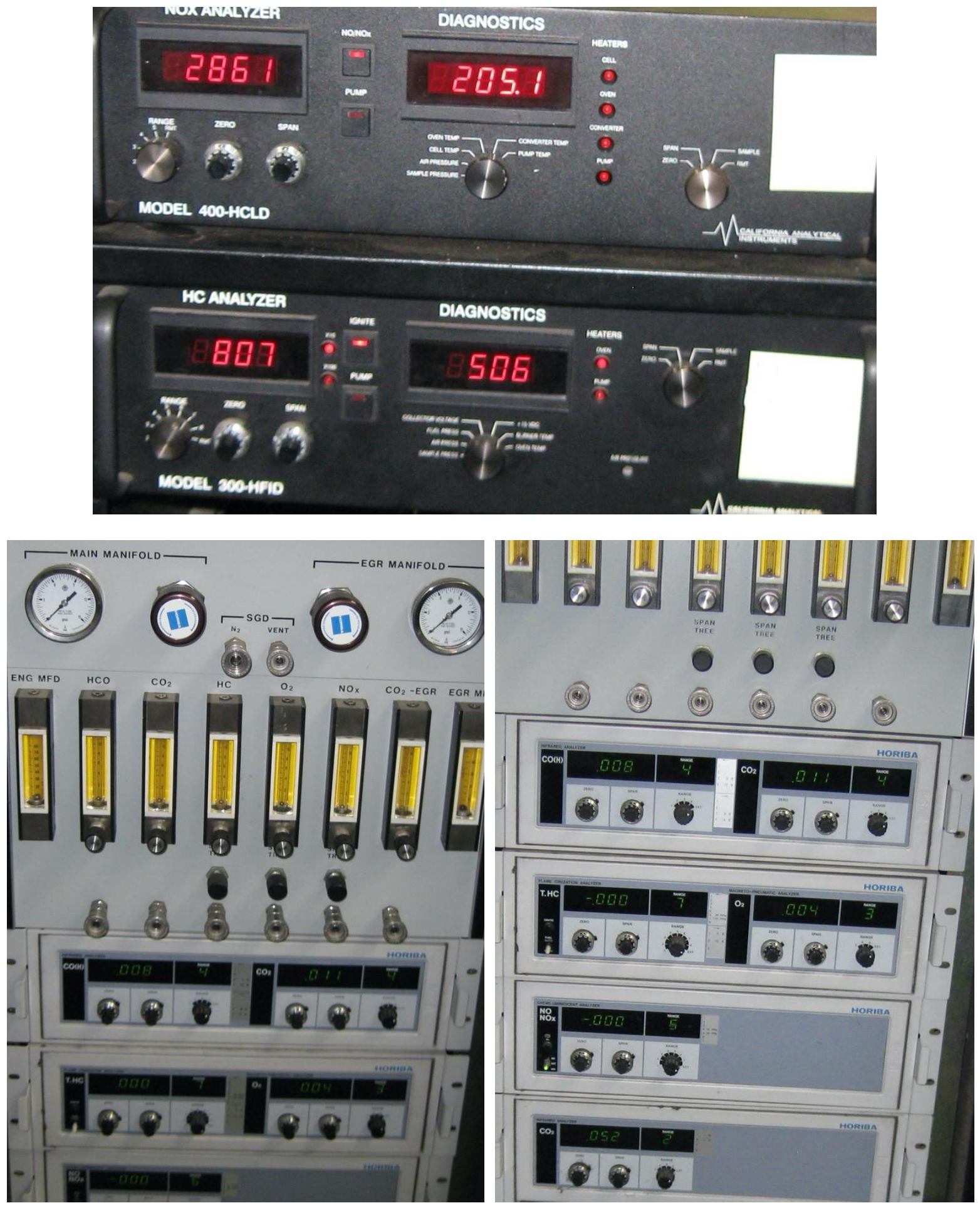

Рисунки 5, 6, 7, - на рисунках показаны примеры фрагментов расположения системы аналитического и контрольно-измерительного оборудования 
испытательного стенда, предназначенного для испытаний и аналитической оченки дизельного двигателя. Очень важно показать и раскрыть новизну применения этой системы, в том числе и контрольно-аналитических компонентов системы с элементами искусственного интеллекта 


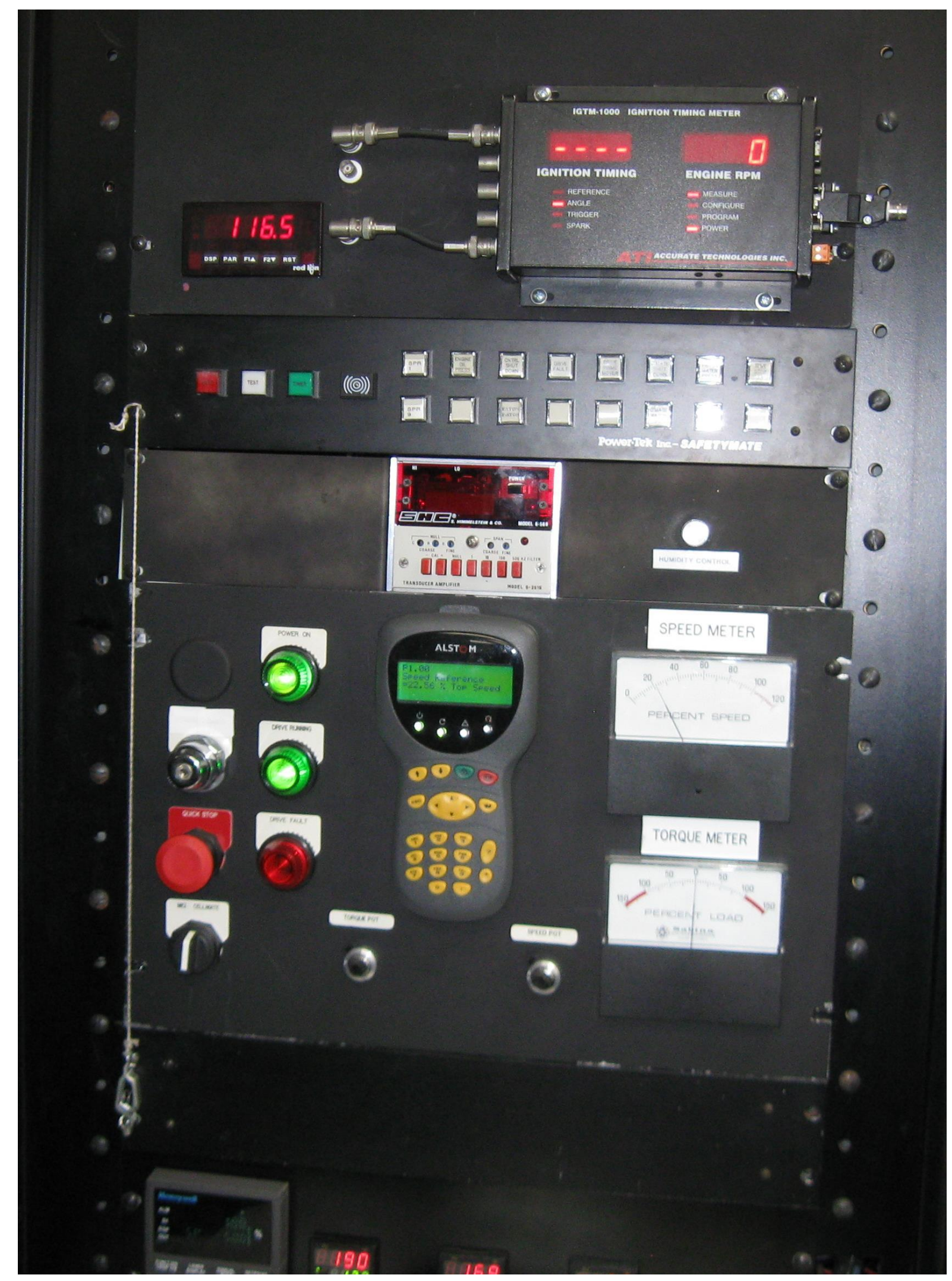

Рисунок 8 
На рисунках 8, 9, 10 и 11 показаны примеры фрагментов расположения системы аналитического и контрольно-измерительного оборудования испытательного стенда, предназначенного для испытаний и аналитической оценки дизельного двигателя. Очень важно показать и раскрыть новизну применения этой системы, в том числе и контрольно-аналитических компонентов системы с элементами искусственного интеллекта

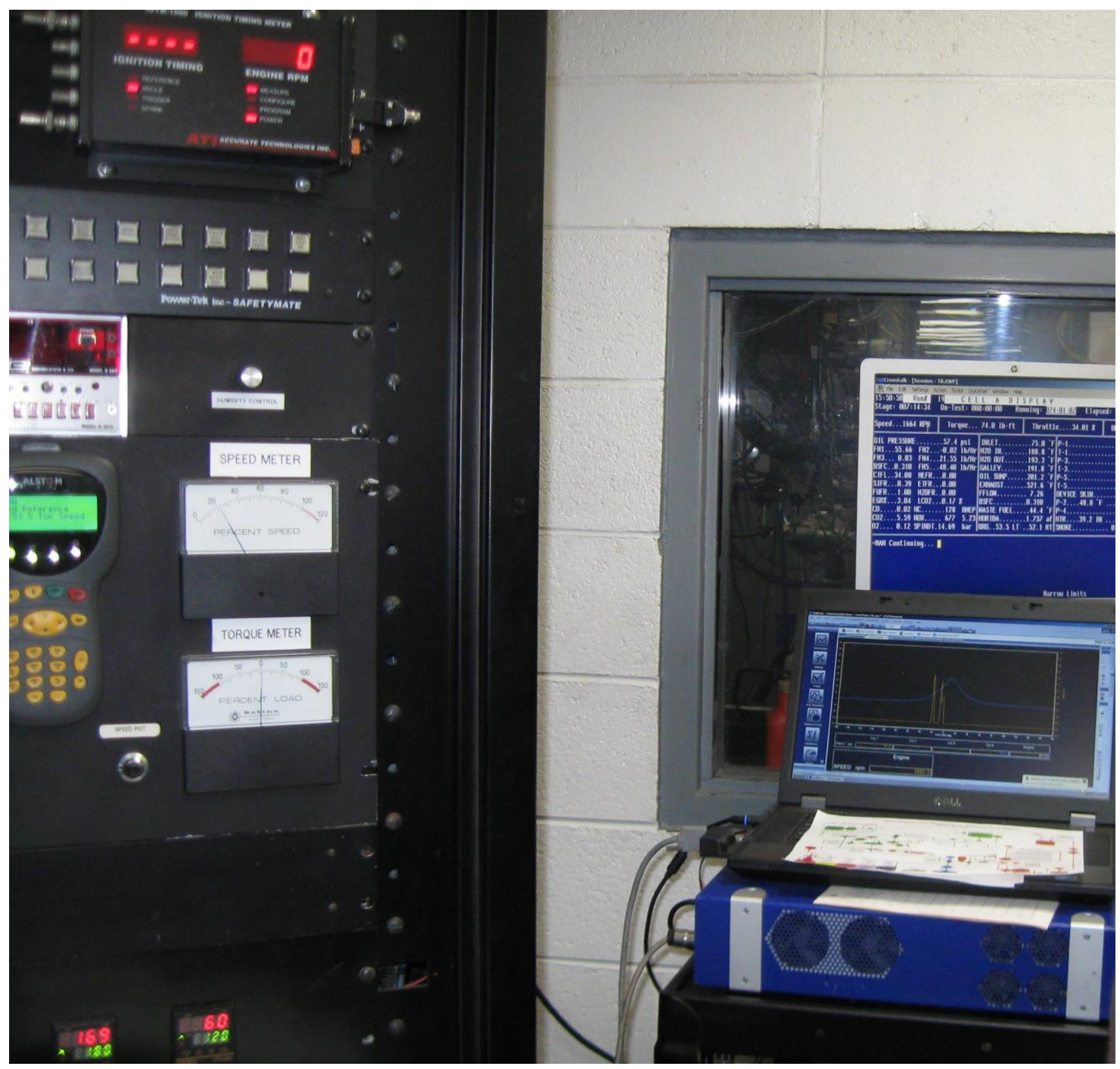

Рисунок 9 


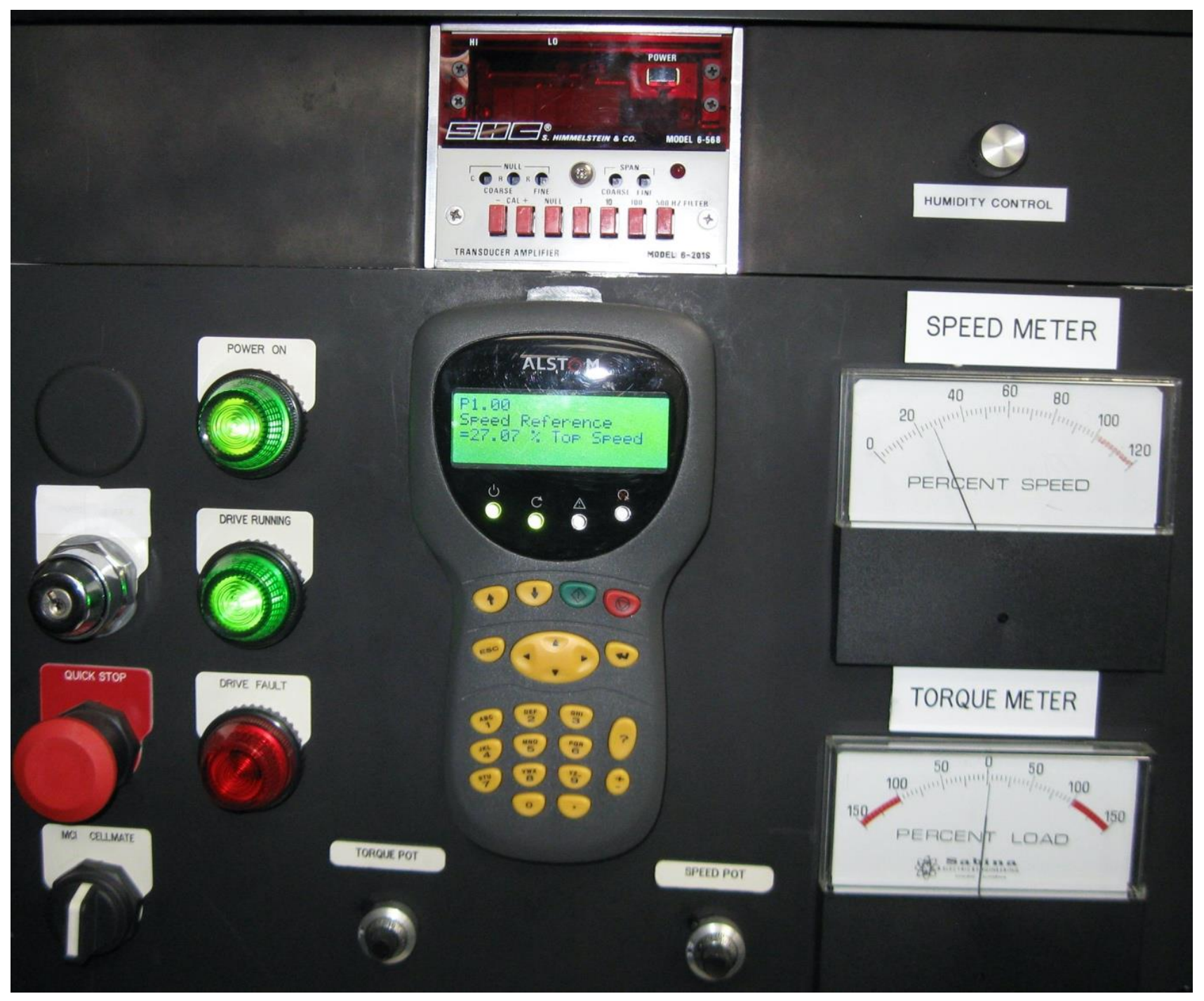

Рисунок 10, - на рисунке показаны в качестве примера компоненты контрольноаналитической системы, позволяющие в прочессе сравнительной эволючии результатов измерений и комплексного мониторинга всего комплекса технологических переходов применять возможности и характеристики элементов искусственного интеллекта и искусственных нейронных сетей

Особенно это важно в постоянной координации основных рабочих параметров испытательного стенда и реакции от этих параметров последующей от испытываемых компонентов двигателя, установленного на динамометрическом столе стенда.

В разделе, в котором приводятся результаты испытаний необходимо связать элементы новизны модифицированных узлов двигателя с определением их реальных показателей и определением влияния этой новизны на характер показателей. 


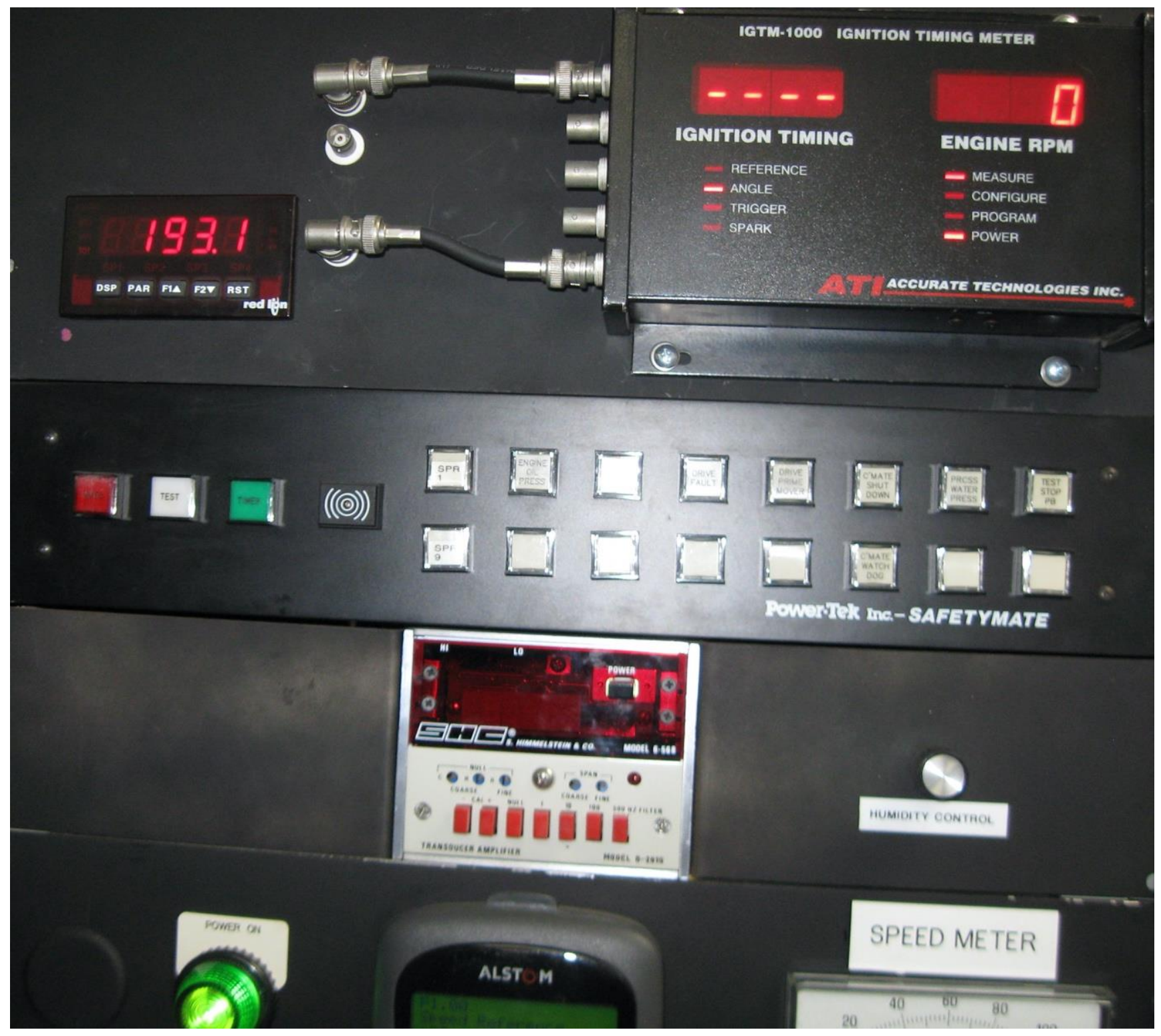

Рисунок 11, - на рисунке также показаны в качестве примера компоненты контрольно-аналитической системы, позволяющче в процессе сравнительной эволюичи результатов измерений и комплексного мониторинга всего комплекса технологических переходов применять возможности и характеристики элементов искусственного интеллекта и искусственных нейронных сетей

В разделе, в котором приводятся результаты испытаний необходимо связать элементы новизны модифицированных узлов двигателя с определением их реальных показателей и определением влияния этой новизны на характер показателей. 


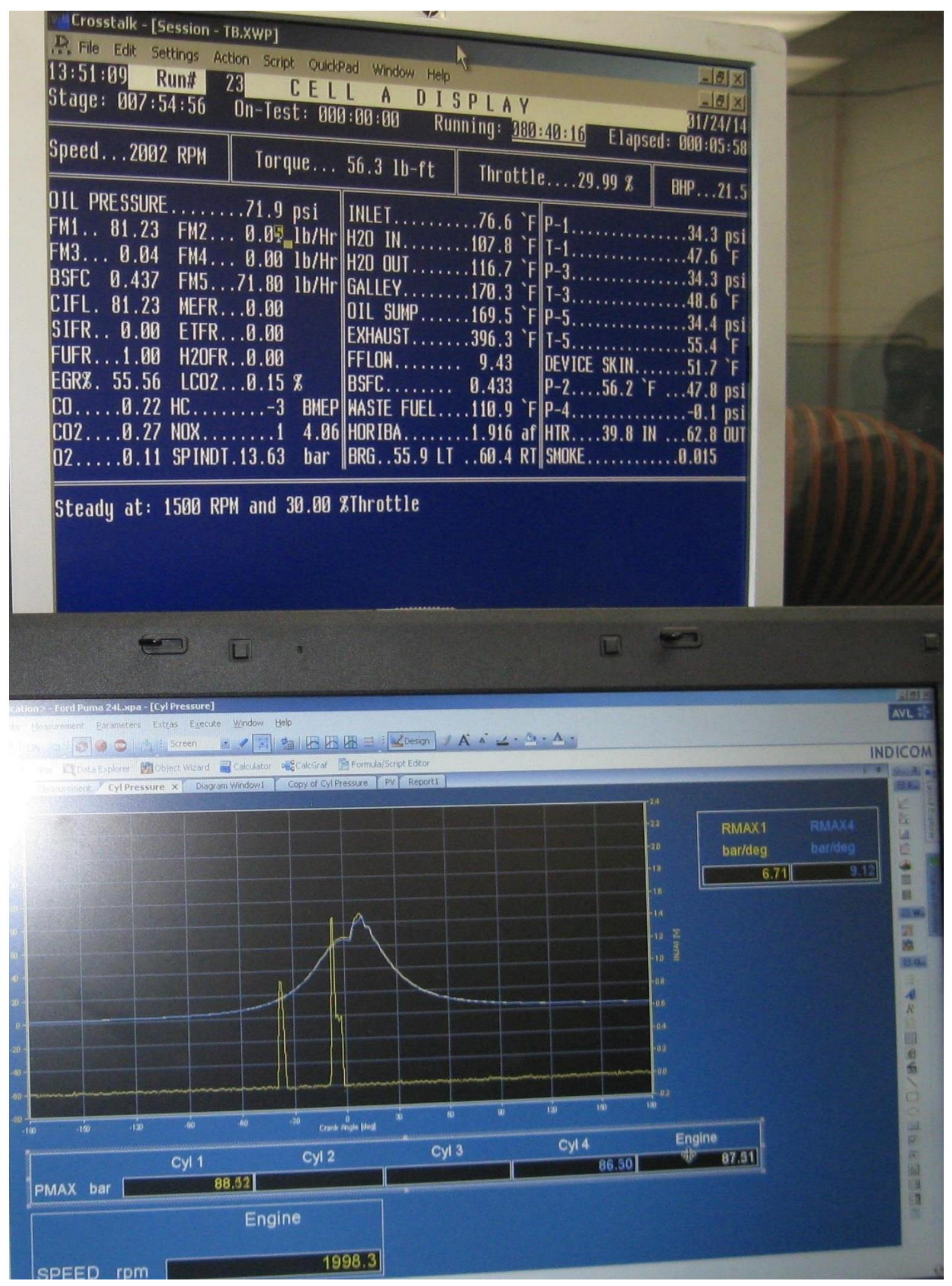

Рисунок 12 


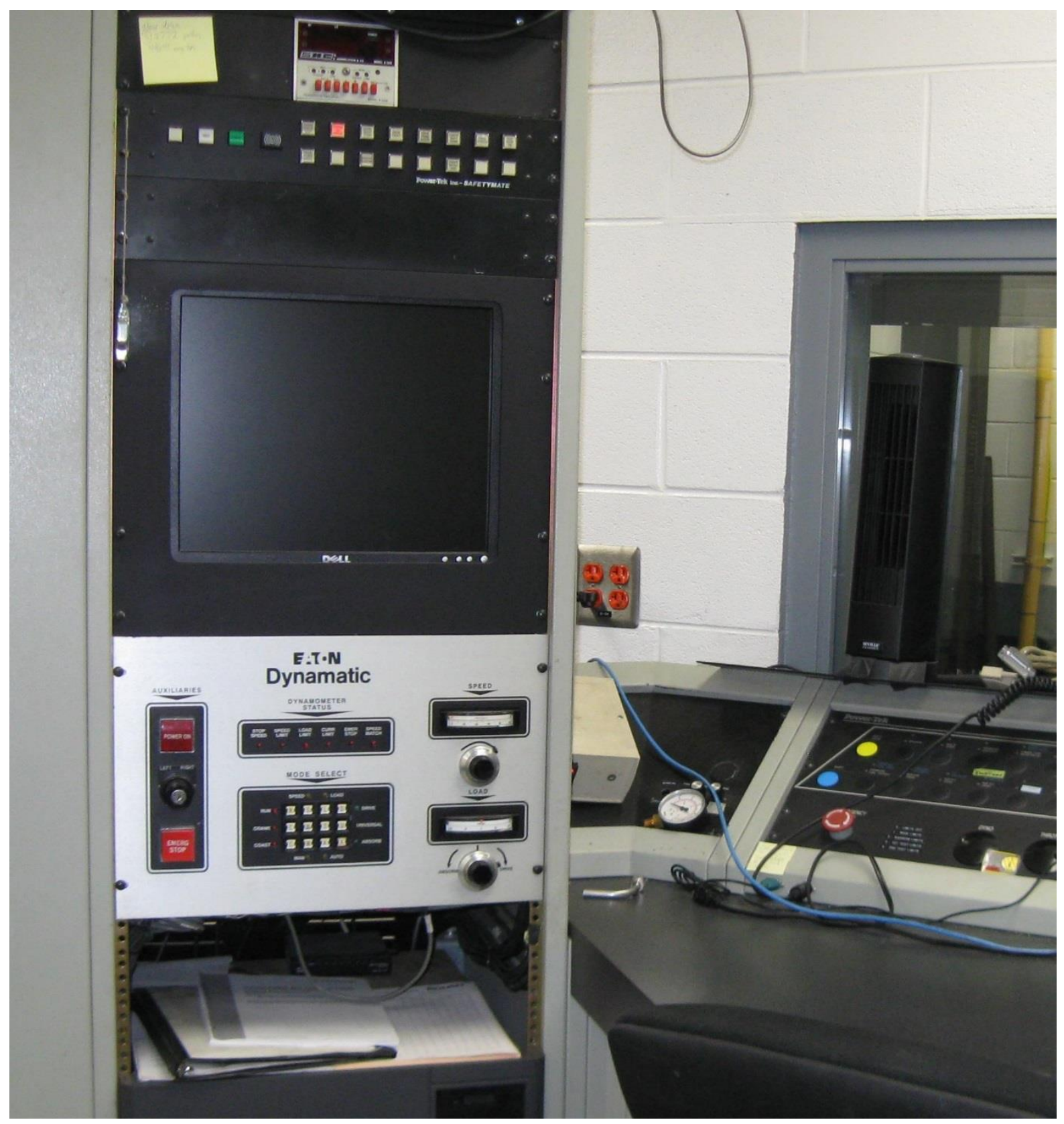

\section{Рисунок 13}

На рисунках 12, 13, 14 и 15 показаны контрольно-измерительные процессы, определяющие характер взаимодействия между различными приборами и системами измерений и их координацией с элементами искусственного интеллекта и искусственными нейронными сетями в части оптимизации основных параметров технической характеристики двигателя, включая и экологические показатели, сопряжённые с формированием топливных смесей, включающих метанол или биологические виды топлива. 


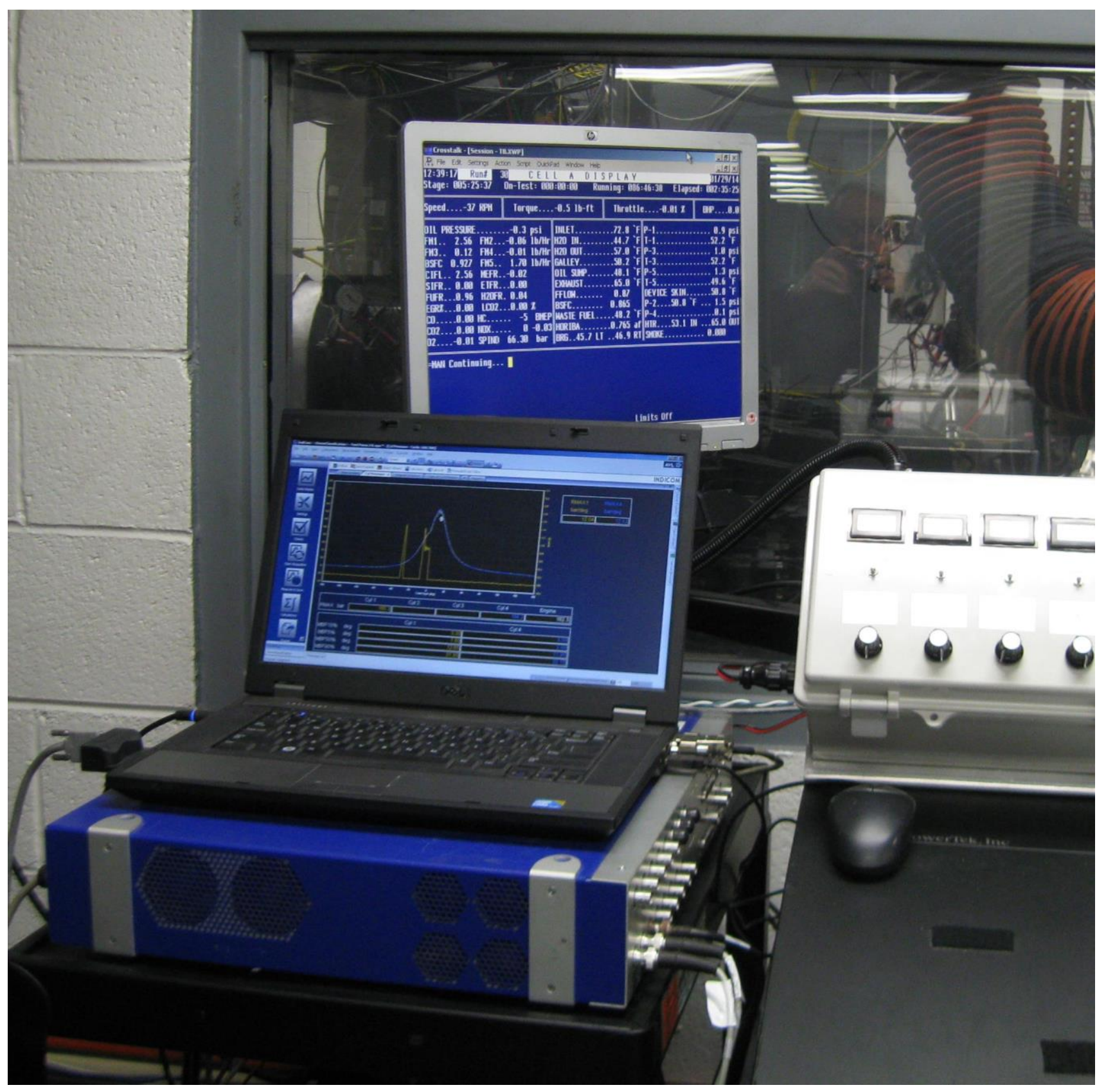

Рисунок 14

Для заявки на предполагаемое изобретение, важно показать влияние элементов и признаков существенной новизны предлагаемого технического решения в их активном сочетании с элементами искусственного интеллекта и искусственными нейронными сетями в сочетании с идеальным конечным результатом направленным на получение необычного результата испытаний, не имеющего места в известных технических решениях и характеризующих предложенное техническое решение. 


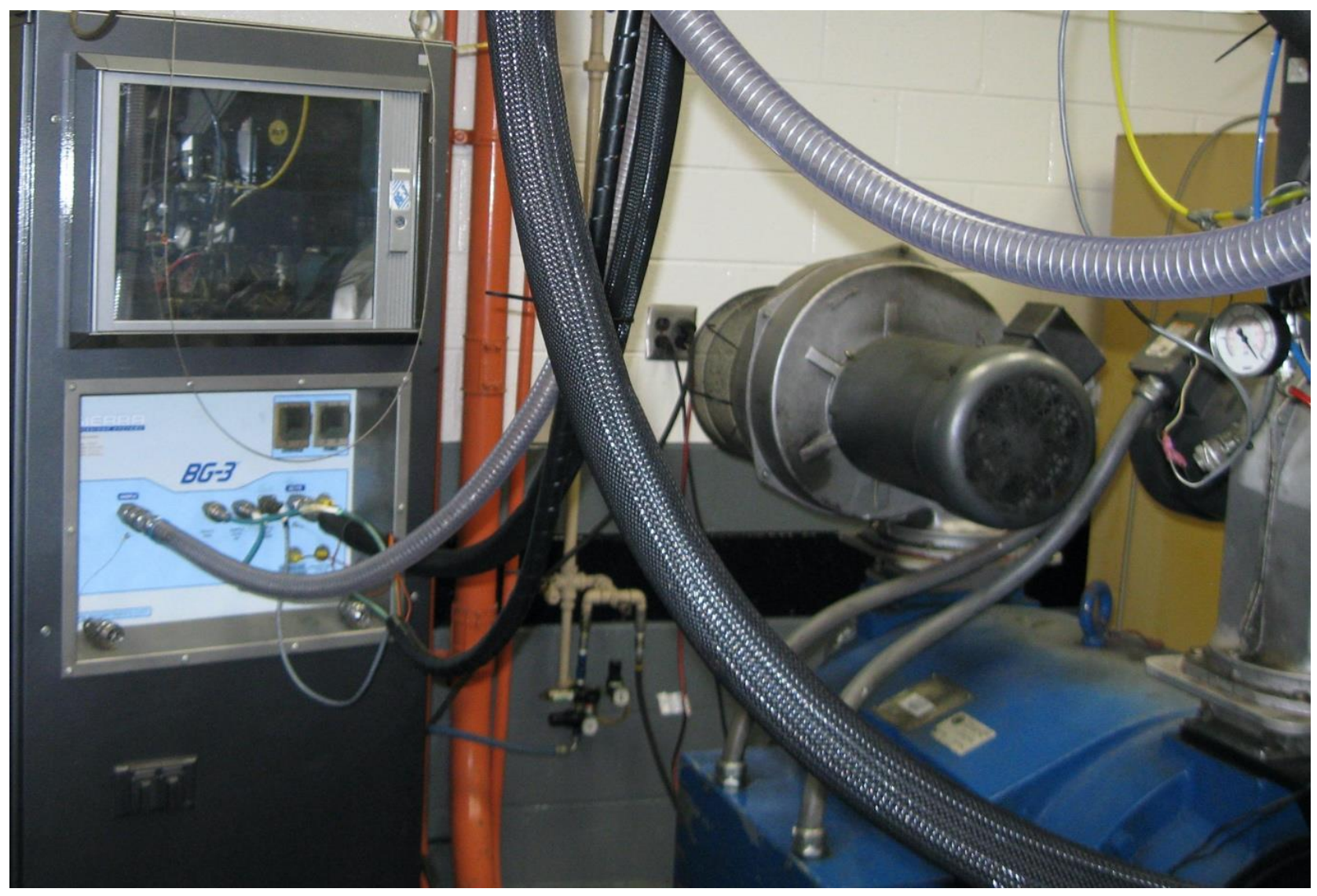

Рисунок 15

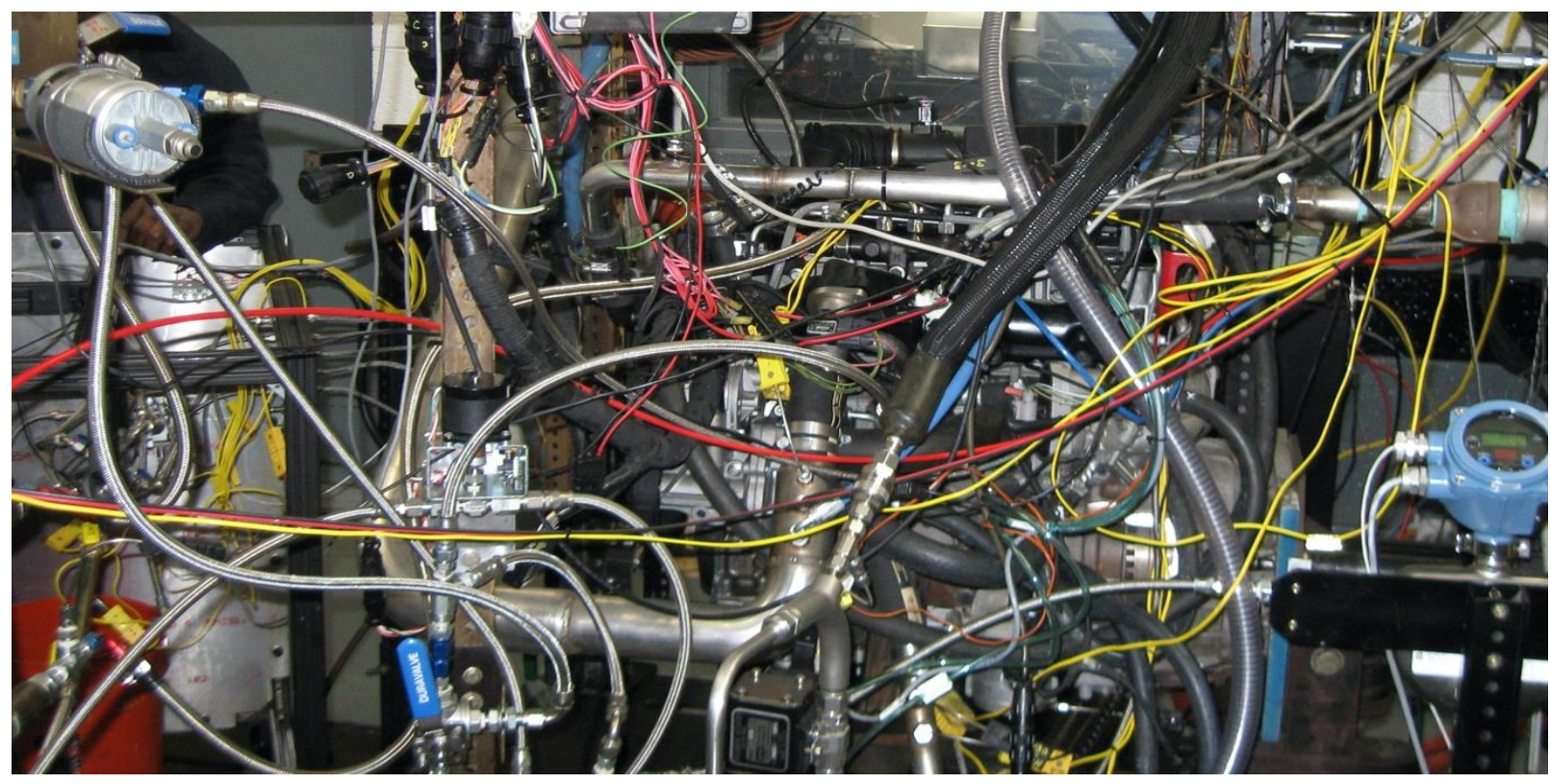

Рисунок 16 
На рисунке 16 показан двигатель на стенде, на котором контролируются более 1200 параметров, обработка результатов измерений которых ведётся в режиме реального времени при помощи элементов искусственного интеллекта и искусственных нейронных сетей.

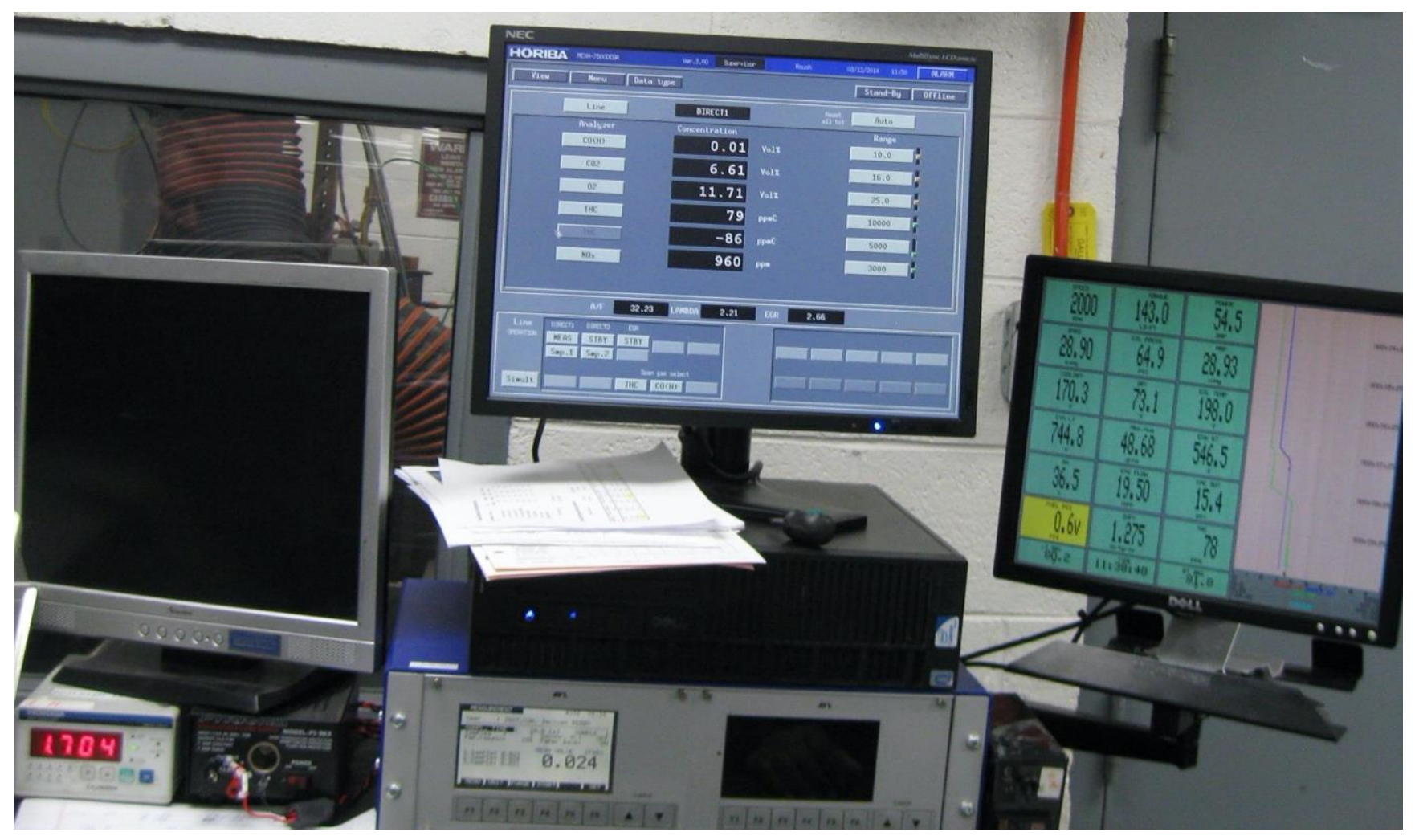

Рисунок 17, - на рисунке показаны в качестве примера компоненты контрольноаналитической системы, позволяющие в процессе сравнительной эволючии результатов измерений и комплексного мониторинга всего комплекса технологических переходов применять возможности и характеристики элементов искусственного интеллекта и искусственных нейронных сетей

Особенно это важно в постоянной координации основных рабочих параметров испытательного стенда и реакции от этих параметров последующей от испытываемых компонентов двигателя, установленного на динамометрическом столе стенда.

В разделе, в котором приводятся результаты испытаний необходимо связать элементы новизны модифицированных узлов двигателя с определением их реальных показателей и определением влияния этой новизны на характер показателей. 


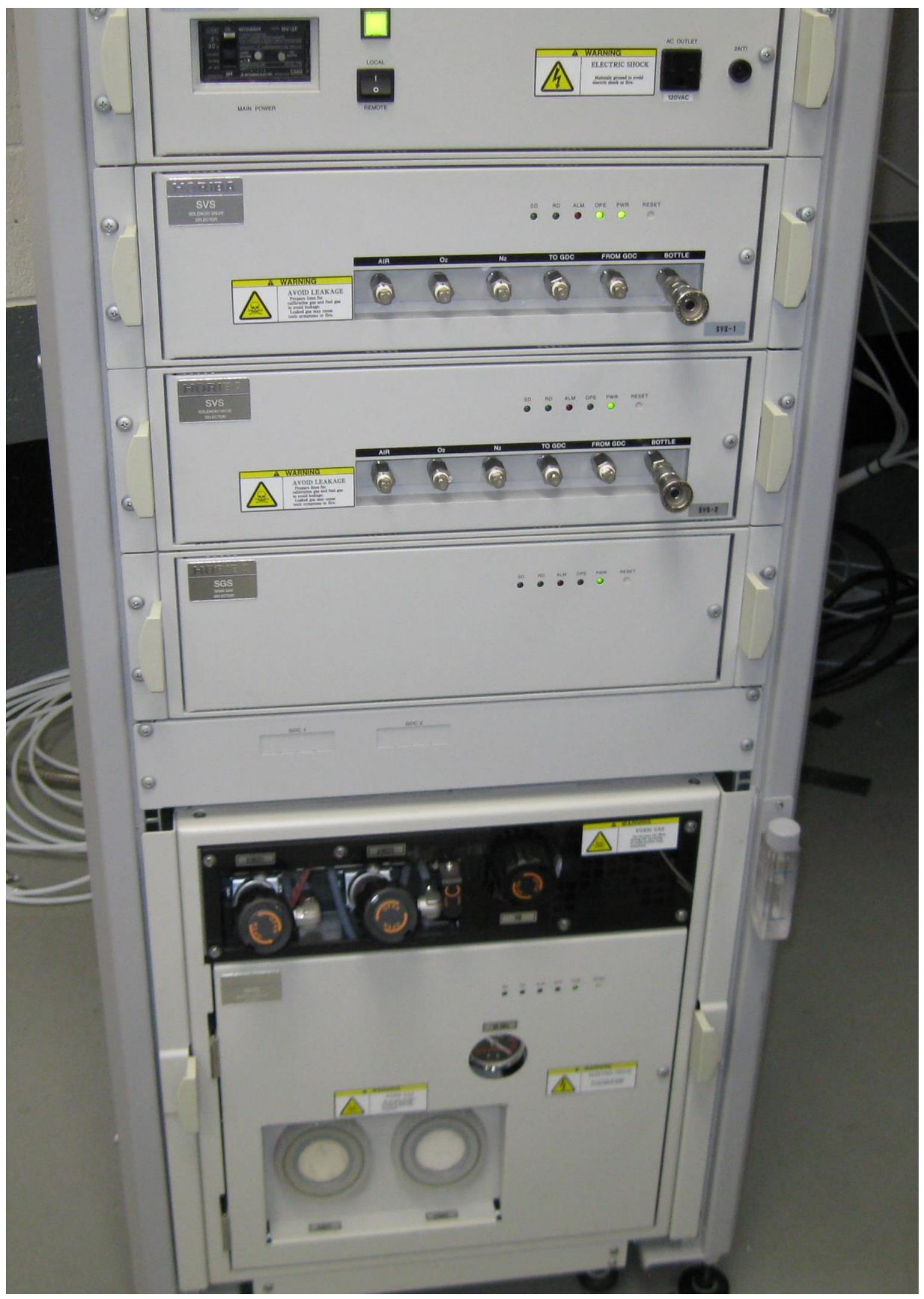

Рисунок 18 


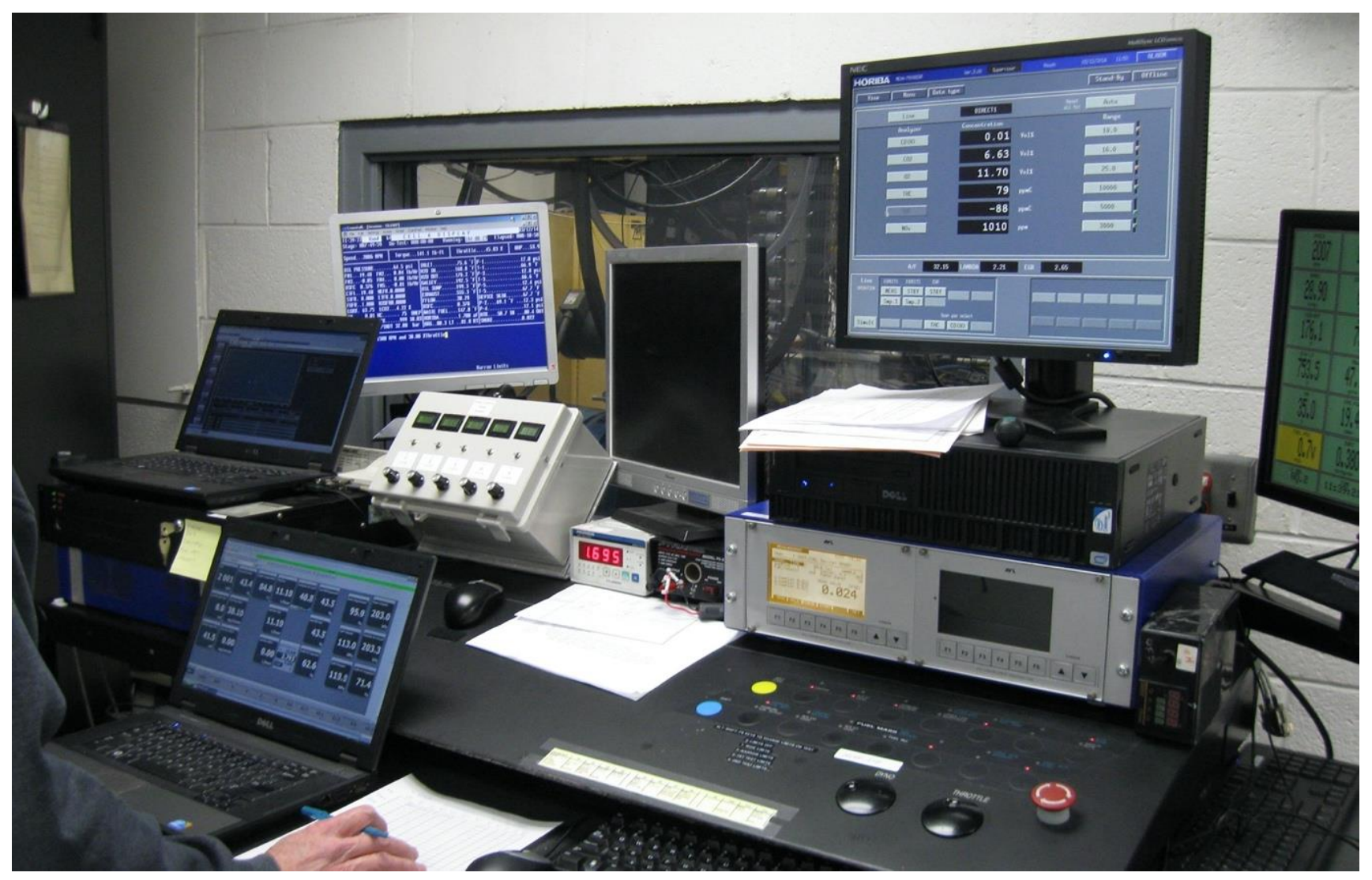

Рисунок 19

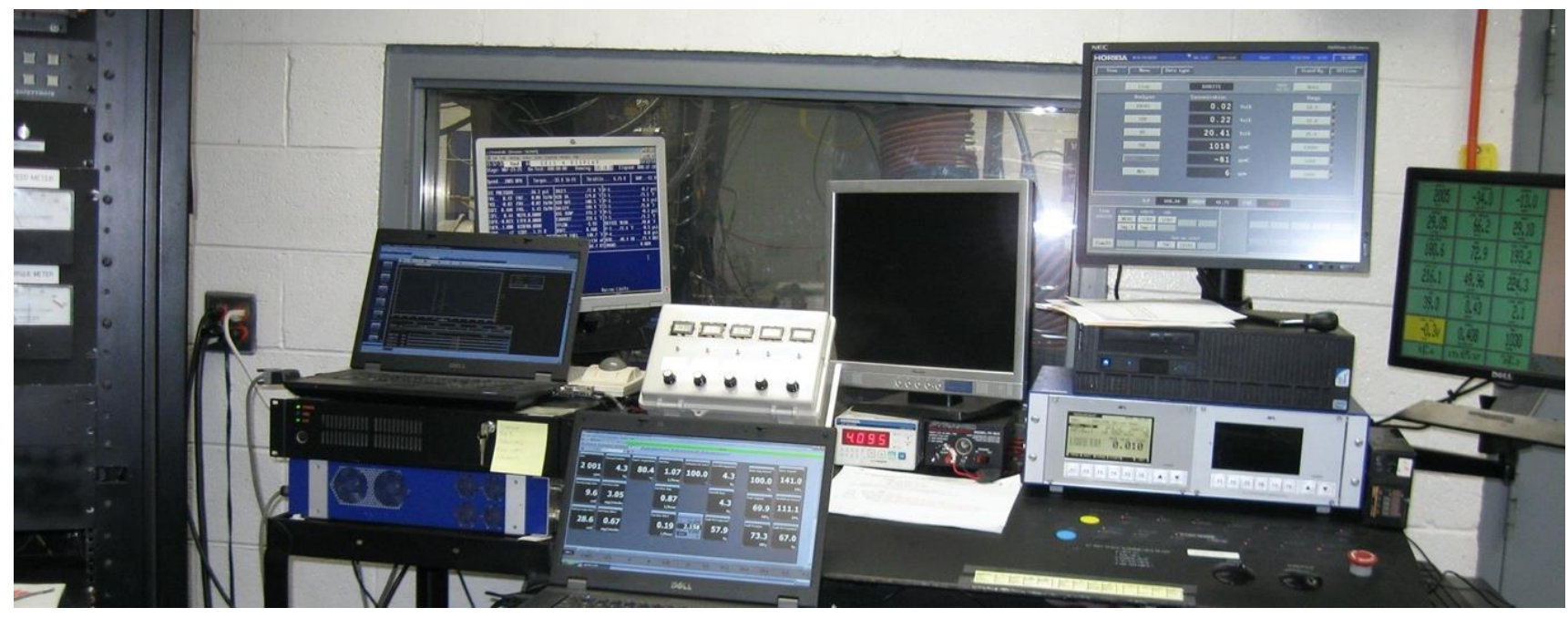

Рисунок 20 


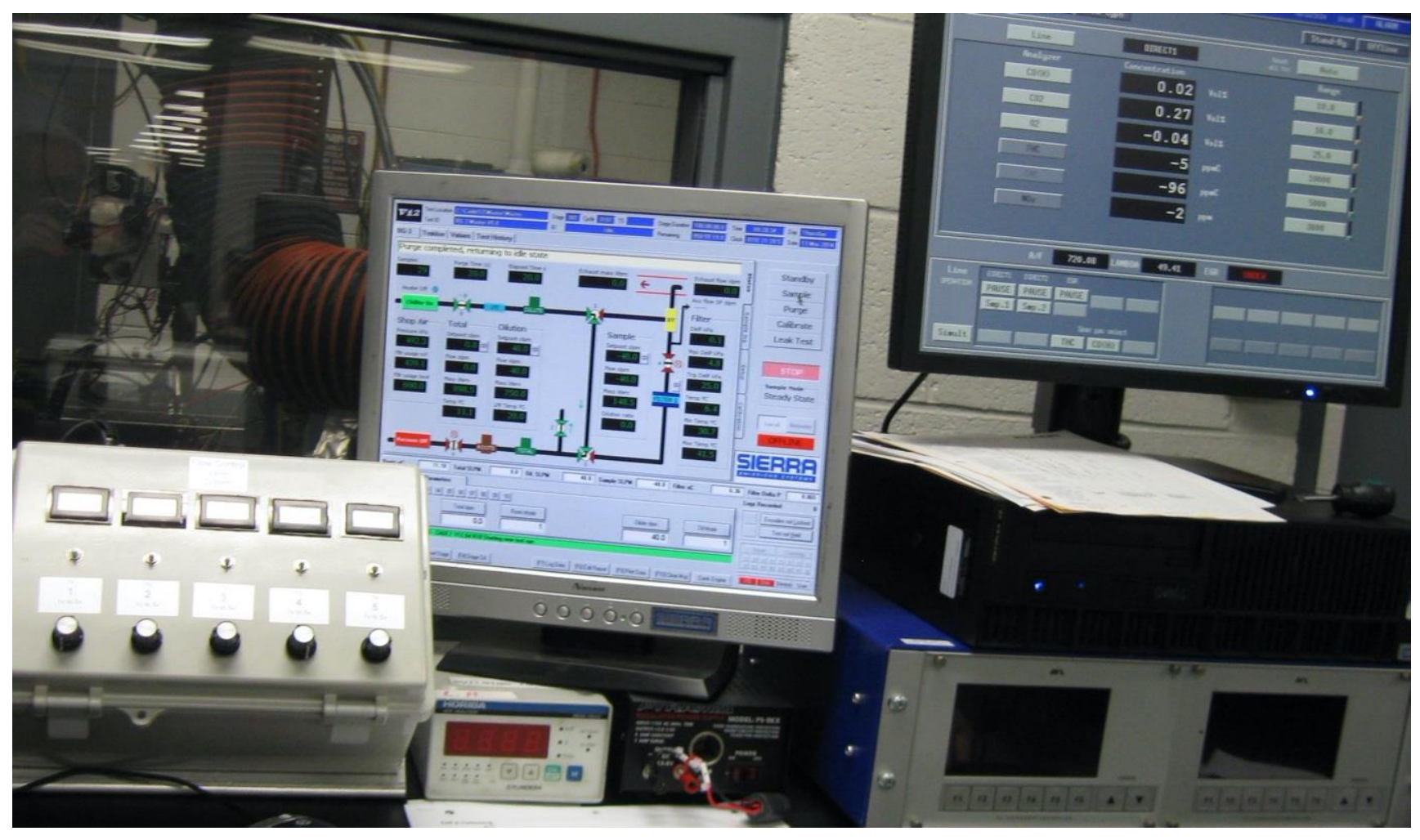

\section{Рисунок 21}

На рисунках 18, 19, 20, 21, 22, 23 и 24, - также показаны в качестве примера связанные функционально между собой и одновременно с элементами искусственного интеллекта и искусственными нейронными сетями компоненты контрольно-аналитической системы, позволяющие в процессе сравнительной эволюции результатов измерений и комплексного мониторинга всего комплекса технологических переходов также комплексно применять возможности и характеристики элементов искусственного интеллекта и искусственных нейронных сетей.

Особенно это важно в постоянной координации основных рабочих параметров испытательного стенда и реакции от этих параметров последующей от испытываемых компонентов двигателя, установленного на динамометрическом столе стенда.

В разделе, в котором приводятся результаты испытаний необходимо связать элементы новизны модифицированных узлов двигателя с определением их реальных показателей и определением влияния этой новизны на характер показателей. 


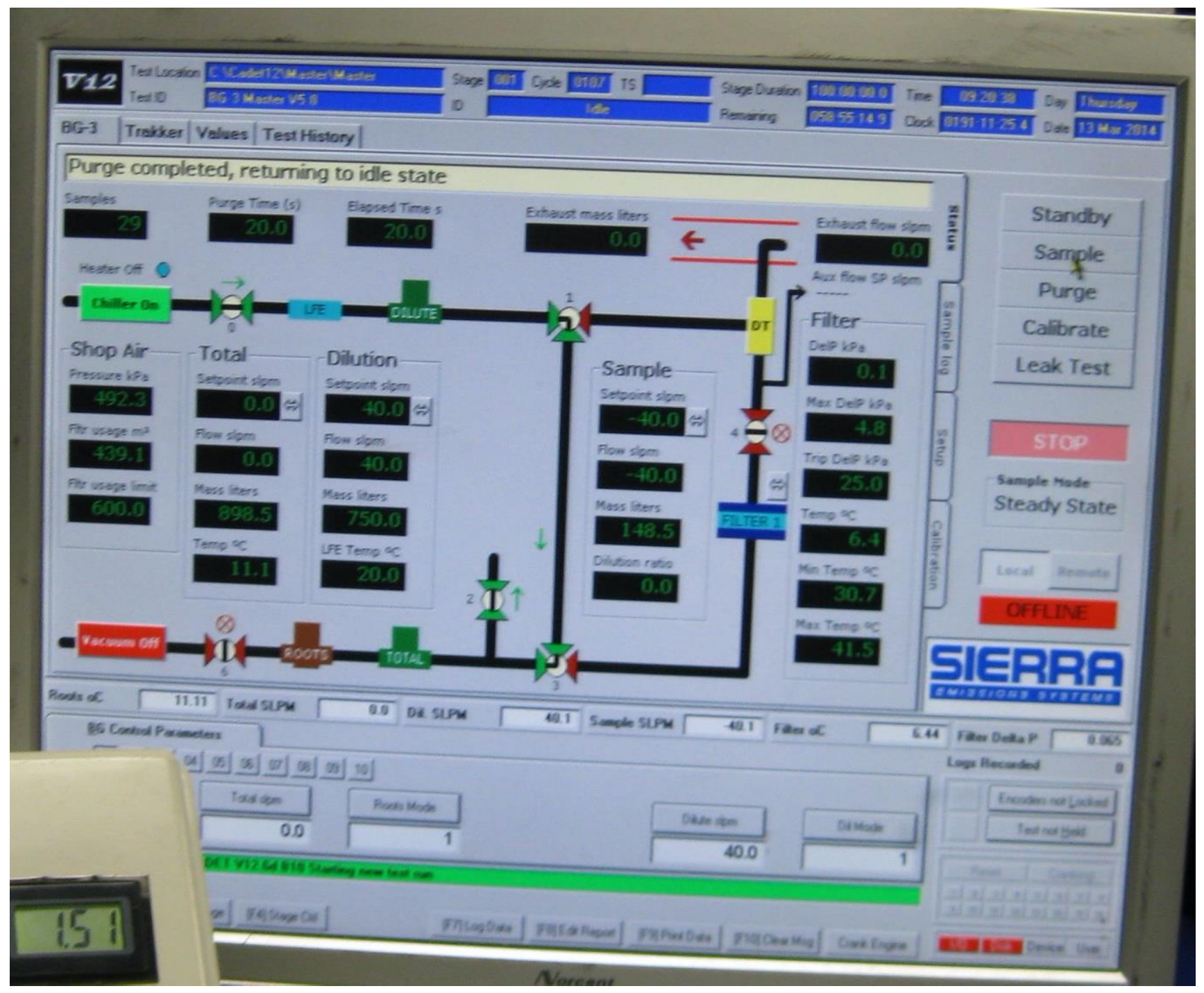

\section{Рисунок 22}

Для приведенного примера характерным являются показатели и сочетания результатов измерений различных, связанных между собой параметров работы двигателя, изменяющихся в зависимости от того фактора, который в максимальной степени влияет на результат, причём это может быть состав топливной смеси, включающий только жидкие компоненты, или жидкие и газообразные компоненты или только газообразные компоненты.

Постоянный анализ результативности всех элементов новизны и их влияния на степень или уровень достижения идеального конечного результата в максимальной степени зависят от включения или не включения в контрольно-аналитический комплекс элементов искусственного интеллекта и искусственных нейронных сетей. 


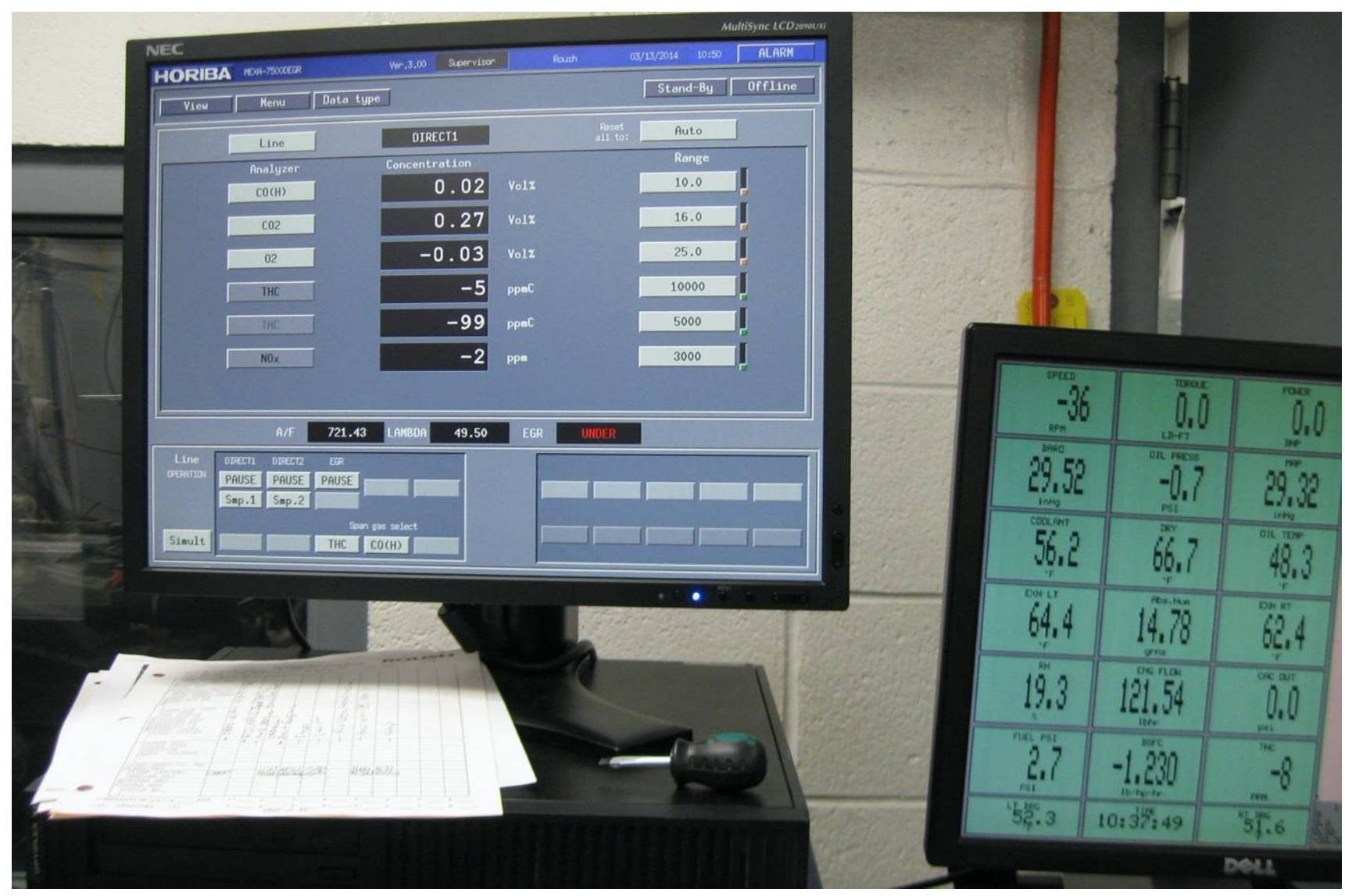

Рисунок 23

Задачей включения и детального анализа влияния от такого рода включения является материал, который должен быть включён в описание заявки на изобретение, в раздел доказательства эффективности элементов существенной новизны и результатов их комплексных испытаний с применением вышеперечисленного оборудования.

В тексте заявки, относящегося к описанию процесса испытаний и характеризации результатов испытаний необходимо дать подробное детальное объяснение принципов построения испытательного процесса в сочетании с аналитической обработкой результатов испытаний и в том числе с связью с требованиями стандартов, действующих в период проведения испытаний.

Описание должно включать характеризацию общих отличительных признаков предполагаемого изобретения и их причинно-следственной связи с полученными результатами.

Также необходимо указать насколько новыми и неочевидными являются полученные результаты. 


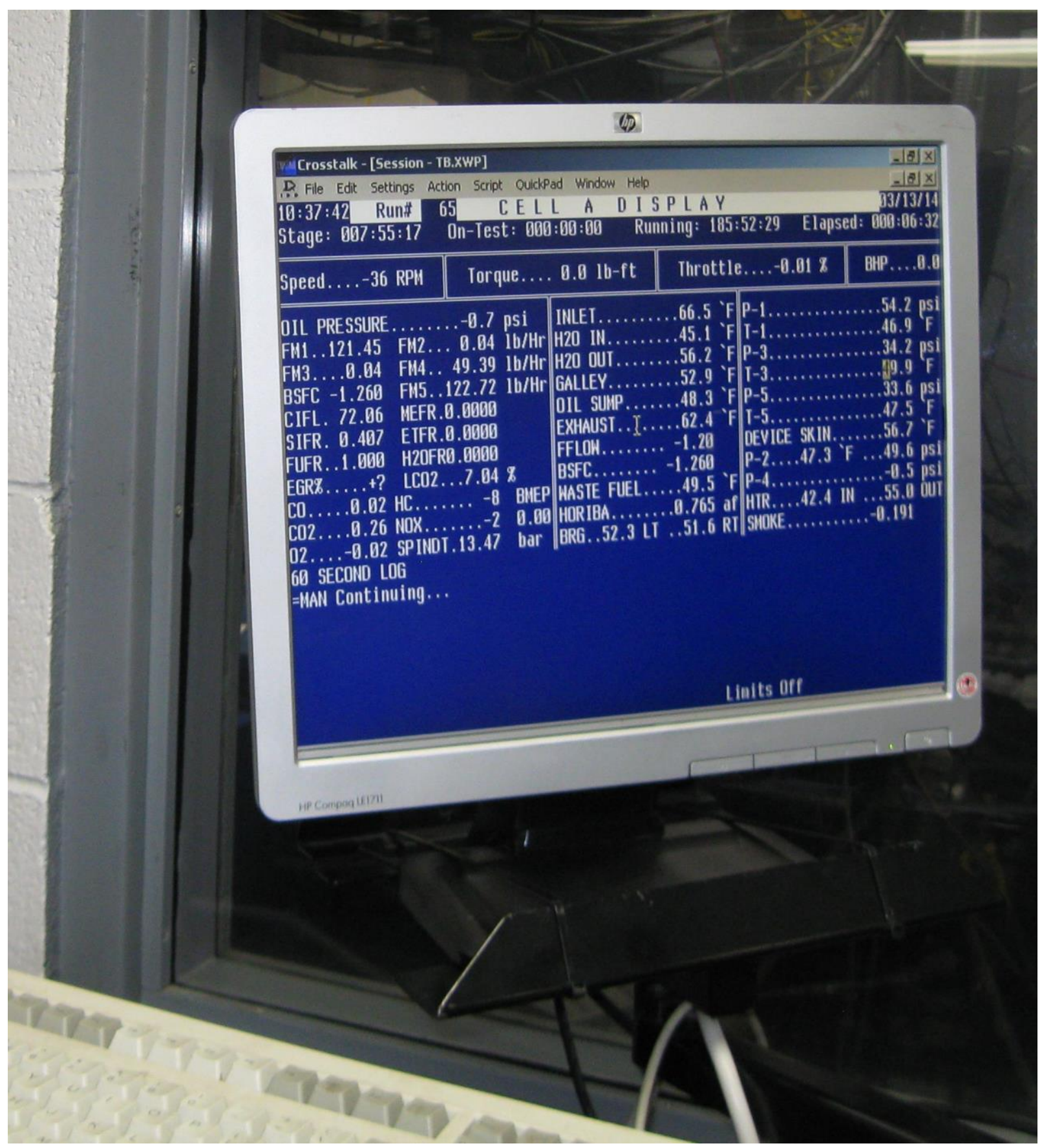

Рисунок 24

Важно показать связь изобретения с умными технологиями и возможностями комбинирования программных продуктов нового решения с элементами конструкции аппарата или системы, формирующими комплекс новых технических решений, положенных в основу предлагаемого изобретения. 
[0085] Test results showed that in the..., - в этом разделе приведенные результаты испытаний идентифицируют и сравнивают с аналогичными результатами испытаний известных устройств или аппаратов.

[0086] В этом разделе доказывают, что полученные результаты испытаний соответствуют ожидаемым и декларируемым показателям идеального конечного результата.

\section{ANALYSIS OF THE TEST RESULTS}

[0087] Qualification test result analysis showed that the embodiments of the invention achieve the objective of..., - в этом разделе приводится структурный анализ и характеризация результатов испытаний опытных образцов изобретённого технического решения.

Далее необходимо проанализировать Общие отличительные признаки изобретения, которые отличают его от известных патентных материалов.

Как пример, рассмотрим изобретение - двигатель внутреннего сгорания, отнесённый к умным транспортным системам по всем элементам конструкции и принципов действия:

○ двигатель внутреннего сгорания, в систему подачи и подготовки топливной смеси которого, интегрировано устройство для динамического смешивания жидкого топлива с другими компонентами топливной смеси;

○ рабочий цикл устройства для динамического смешивания жидкого топлива с другими компонентами топливной смеси происходит в топливном трубопроводе при основных параметрах работы топливного трубопровода, которые имеют место в обычном рабочем цикле любого двигателя внутреннего сгорания;

○ в работе двигателя с интегрированным устройством для динамического смешивания жидкого компонента топливной смеси с другими компонентами топливной смеси используются только материалы и вещества, которые принято использовать при работе серийных двигателей внутреннего сгорания;

○ устройство для динамического смешивания компонентов имеет два последовательно расположенных по ходу движения топлива комплексных вводных устройств, завершающихся вихревыми генераторами, формирующими в пределах топливного трубопровода вихревую трубу; 
○ устройство для динамического смешивания компонентов топливной смеси имеет систему смешивания, которая при смешивании уравнивает уровень турбулентности в каждом поперечном сечении топливного трубопровода в центре поперечного сечения трубопровода и на его периферии;

○ топливная смесь на выходе из устройства динамического смешивания компонентов топливной смеси, в зависимости от пропорции смешивания между компонентами, и в зависимости от характера и вида компонентов, может иметь различную структуру и состав:
○ эмульсии
○ вспененной эмульсии
○ вспененной топливной смеси
○ топливной пены
○ аэрозоли
○ газированного топлива.

Ни в одном из известных технических решений топливная смесь перед впрыском в цилиндр или камеру сгорания не имеет такой состав и структуру.

В случае, если в качестве компонента топливной смеси выступает газ, вследствие того, что в устройстве для динамического смешивания жидкого топлива с другими компонентами топливной смеси площадь контактной поверхности в пограничном слое - жидкость/газ увеличивается по сравнению с противопоставленными техническими решениями по крайней мере на порядок (как минимум в 10 раз) концентрация растворённого газа в жидкости увеличивается пропорционально увеличению площади контакта:

○ для смешивания применяются только материалы и компоненты, как жидкие так и газообразные, которые традиционно применяются для приготовления топливных смесей;

○ для приготовления топливных смесей в предлагаемом техническом решении применяются только известные технологические приёмы и действия..., только гидродинамическое и аэродинамическое смешивание и не применяются технологические приёмы и действия, как в известных патентных аппликациях:

○ «Fuel converter», - декларируемый эффект достигается за счёт нагрева, испарения и катализа топливной смеси, а не за счёт только гидродинамического и аэродинамического смешивания, как имеет место в предложенном техническом решении. В известном материале отсутствуют признаки присущие техническому решению, положенному в основу настоящего изобретения и, исходя из этого, 
известное техническое решение не является техническим эквивалентом заявленного технического решения ни по одному из признаков и критериев.

○ «Heated catalyzed fuel injector for injection ignition engines», декларируемый эффект достигается за счёт дополнительного сжатия и нагрева топливной смеси и дополнительного каталитического воздействия на топливную смесь, а не за счёт только гидродинамического и аэродинамического смешивания компонентов топливной смеси, как в предложенном техническом решении. В известном материале отсутствуют признаки присущие техническому решению, положенному в основу настоящего изобретения и, исходя из этого известное техническое решение не является техническим эквивалентом заявленного технического решения ни по одному из признаков и критериев.

○ «Single nozzle direct injection system for rapidly variable gasoline/antiknock agents mixtures», - декларируемый эффект является абсолютно отличающимся от эффекта у предложенного технического решения. Цель у известного технического решения..., - смешивание с топливной смесью антидетонационного агента, который снижает уровень детонации бензина. При этом смешивание осуществляется по технологии, абсолютно не равной и не эквивалентной ни по одному из признаков заявленному техническому решению; В известном материале отсутствуют признаки присущие техническому решению, положенному в основу настоящего изобретения, и, исходя из этого противопоставленное техническое решение не является техническим эквивалентом заявленного технического решения ни по одному из признаков и критериев.

○ «Internal combustion engine/water sours system», - декларируемый эффект - охлаждение выхлопных газов с целью конденсации из них воды, предлагается осуществить при помощи сжатия выхлопных газов и формирования из них вихревой трубы. Исходя из материалов, изложенных в известном техническом решении, можно сделать вывод о невозможности в этом техническом решении снизить температуру выхлопных газов до такого уровня, когда возможна конденсация воды; Известное техническое решение абсолютно не работоспособно. В известном материале отсутствуют признаки присущие техническому решению, положенному в основу настоящего изобретения и, исходя из этого известное техническое решение не является техническим 
эквивалентом заявленного технического решения ни по одному из признаков и критериев.

Исходя из результатов испытаний прототипа предложенного технического решения интегрированного в топливную систему серийного дизельного двигателя, полученный результат является новым для реальной практики эксплуатации дизельных двигателей и составляет, $-97 \%$ снижения уровня сажи, образовавшейся в результате горения топливной смеси в цилиндрах двигателя.

Такой результат является абсолютно новым как для дизельных так и для бензиновых двигателей, и ни одно из известных технических решений ни в одном из своих признаков, не решает эквивалентной технической задачи на таком уровне, а это значит, что известные технические решения по отношению к предложенному техническому решению $\mathrm{c}$ доказанной полной работоспособностью и, подтверждённой документально, эффективностью, для всех известных четырёх технических решений с неопределённой работоспособностью и с не доказанной эффективностью является с технической точки зрения неправомочным. Кроме того, предотвращение образования сажи в цилиндрах двигателя за счёт только гидродинамического и аэродинамического смешивания стандартных компонентов топливной смеси, не известного по предыдущим публикациям и изобретениям и также отсутствующего в известных технических решениях, является по отношению к каждому из, известных технических решений, отдельно и всем противопоставленным техническим решениям в совокупности, абсолютно новым.

\section{EXAMPLES OF APPLICATIONS}

[0088] In some embodiments..., - в этом разделе приводятся примеры различных вариантов применения изобретения или различных вариантов исполнения изобретения, если таковые имеются ; если разнообразие вариантов исполнений и множество вариантов применения не вошли в основные отличительные признаки формулы изобретения, то очень важно, что бы эти варианты были как можно более чётко и понятнее детально описаны. В дальнейшем при идентификации и проверке факта действительного использования изобретения, эти образцы и примеры применения помогают более убедительно доказать факты использования изобретения.

[0089] In some embodiments..., - в этом разделе приводятся примеры различных вариантов применения изобретения или различных вариантов исполнения 
изобретения, если таковые имеются; если разнообразие вариантов исполнений и множество вариантов применения не вошли в основные отличительные признаки формулы изобретения, то очень важно, что бы эти варианты были как можно более чётко и понятнее детально описаны. В дальнейшем при идентификации и проверке факта действительного использования изобретения, эти образцы и примеры применения помогают более убедительно доказать факты использования изобретения.

[0090] In some embodiments..., - в этом разделе указывается благодаря каким оригинальным признакам и сочетаниям признаков в каждом из рассмотренных вариантов, обеспечивается достижение идеального конечного результата, ожидаемое именно от этого исполнения.

[0091] In some embodiments..., - в этом разделе указывается благодаря каким оригинальным признакам и сочетаниям признаков в каждом из рассмотренных вариантов, обеспечивается достижение идеального конечного результата, ожидаемое именно от этого исполнения.

[0092] In some embodiments..., - в этом разделе указывается благодаря каким оригинальным признакам и сочетаниям признаков в каждом из рассмотренных вариантов, обеспечивается достижение идеального конечного результата, ожидаемое именно от этого исполнения.

[0093] In some embodiments..., - в этом разделе указывается благодаря каким оригинальным признакам и сочетаниям признаков в каждом из рассмотренных вариантов, обеспечивается достижение идеального конечного результата, ожидаемое именно от этого исполнения.

[0094] In some embodiments..., - в этом разделе указывается благодаря каким оригинальным признакам и сочетаниям признаков в каждом из рассмотренных вариантов, обеспечивается достижение идеального конечного результата, ожидаемое именно от этого исполнения.

[0095] In some embodiments..., - в этом разделе указывается благодаря каким оригинальным признакам и сочетаниям признаков в каждом из рассмотренных вариантов, обеспечивается достижение идеального конечного результата, ожидаемое именно от этого исполнения.

[0096] In some embodiments..., - в этом разделе указывается благодаря каким оригинальным признакам и сочетаниям признаков в каждом из рассмотренных вариантов, обеспечивается достижение идеального конечного результата, ожидаемое именно от этого исполнения. 
[0097] In some embodiments..., - в этом разделе указывается благодаря каким оригинальным признакам и сочетаниям признаков в каждом из рассмотренных вариантов, обеспечивается достижение идеального конечного результата, ожидаемое именно от этого исполнения.

[0098] In some embodiments..., - в этом разделе указывается благодаря каким оригинальным признакам и сочетаниям признаков в каждом из рассмотренных вариантов, обеспечивается достижение идеального конечного результата, ожидаемое именно от этого исполнения.

[0099] An operating principle of the devices is..., - в этом разделе анализируются и сравниваются варианты оперирования аппаратом или устройством, анализируются варианты процессов управления, контроля и применение принципов формирования элементов обратной связи между исполнительными механизмами и системами или процессорами управления.

[0100] В этом разделе сравниваются возможности и особенности различных, перечисленных в разделе вариантов и примеров применения изобретения.

[0101] In some embodiments..., - в этом разделе указывается благодаря каким оригинальным признакам и сочетаниям признаков в каждом из рассмотренных вариантов, обеспечивается достижение идеального конечного результата, ожидаемое именно от этого исполнения.

[0102] In some embodiments..., - в этом разделе указывается благодаря каким оригинальным признакам и сочетаниям признаков в каждом из рассмотренных вариантов, обеспечивается достижение идеального конечного результата, ожидаемое именно от этого исполнения.

[0103] It will be appreciated that the..., - в этом разделе указывается благодаря каким оригинальным признакам и сочетаниям признаков в каждом из рассмотренных вариантов, обеспечивается достижение идеального конечного результата, ожидаемое именно от этого исполнения.

[0104] Various embodiments of the invention allow one or more of the following advantages..., - в этом разделе указываются и анализируются любые положительные результаты возникшие при реализации изобретения.

[0105] It will be appreciated by those skilled in the art that various omissions, additions and modifications may be made to the methods and structures described above without departing from the scope of the invention. All such modifications and changes are intended to fall within the scope of the invention, as defined by the appended claims. 
Как пример аналитической оценки, приводим сравнительную Характеристику инновационного продукта, который в общем можно представить как, например топливную смесь, заключается в следующем:

○ продукт формируется в течение короткого промежутка времени - это время может быть равным как минимум нескольким миллисекундам, за которые продукт получает все необходимые характеристики и свойства и сразу становится пригодным к дальнейшему применению;

○ продукт сохраняет свои свойства в течении длительного времени, если давление, под которым он сформирован не изменяется или если оно увеличивается;

○ продукт способен сохранить свои свойства и характеристики и при резком повышении давления (при испытаниях получено давление продукта до 2000 бар и при этом никаких изменений в структуре и свойствах продукта не обнаружено);

○ продукт имеет гибкую гидродинамическую структуру;

○ продукт имеет гибкую химическую структуру;

○ продукт может состоять полностью из однородных по свойствам жидкостей и газов, при этом все жидкие и газообразные компоненты могут быть смешаны в различных пропорциях и сочетаниях;

○ продукт может состоять из неоднородных по свойствам жидкостей и газов, при этом все жидкие и газообразные компоненты могут быть смешаны в различных пропорциях и сочетаниях;

○ к продукту после формирования могут быть добавлены различные химические вещества, не входящие в состав исходного продукта;

○ к продукту во время формирования могут быть добавлены различные химические вещества, не входящие в состав исходного продукта.

Изобретённый продукт может иметь множество разновидностей. К разновидностям изобретённого продукта можно отнести:

○ гомогенную смесь, состоящую из, как минимум, двух однородных жидкостей;

○ гомогенную смесь, состоящую из, как минимум, двух разнородных жидкостей;

○ смесь, состоящую, как минимум, из двух однородных жидкостей и, как минимум, одного газа;

○ смесь, состоящую, как минимум, из двух разнородных жидкостей и, как минимум одного газа; 
○ смесь, состоящую из, как минимум, одного раствора из жидкостей и, как минимум, одной дополнительной жидкости;

○ смесь, состоящую, как минимум, из двух однородных растворов жидкостей;

○ смесь, состоящую, как минимум из двух разнородных растворов жидкостей;

○ смесь, состоящую, как минимум, из двух однородных растворов жидкостей и, как минимум, одного газа;

○ смесь, состоящую, как минимум, из двух разнородных растворов жидкостей и, как минимум, одного газа.

Химические реагенты могут вводиться в смесь как во время формирования, так и после формирования. Такие типы смеси также могут иметь множество разновидностей:

○ смесь, состоящую из, как минимум, двух однородных жидкостей с добавлением после формирования, дополнительных химических реагентов;

○ смесь, состоящую из, как минимум, двух разнородных жидкостей с добавлением после формирования, дополнительных химических реагентов;

○ смесь, состоящую из, как минимум, одного раствора жидкостей и, как минимум, одной жидкости с добавлением после формирования, дополнительных химических реагентов;

○ смесь, состоящую, как минимум, из двух однородных растворов жидкостей с добавлением после формирования, дополнительных химических реагентов;

○ смесь, состоящую, как минимум из двух разнородных растворов жидкостей с добавлением после формирования, дополнительных химических реагентов.

Далее необходимо раскрыть суть технологии, которая положена в основу формирования нового продукта, - в качестве примера приведём смесь, суть технологии производства которой состоит в следующем:

○ в момент формирования смеси уровень турбулентности во всех точках сечения потоков базовых компонентов смеси уравнивается, за счёт чего достигается необходимый уровень равномерности состояния по всему объёму и необходимый уровень размеров частиц компонентов смеси;

○ кинетическая энергия частиц всех компонентов смеси консервируется, и эта энергия высвобождается в том случае, если давление, под которым находится смесь снижается или вообще становится равным атмосферному;

○ высвобождение кинетической энергии компонентов смеси помогает (как например в процессе горения получить максимум распыления топливной жидкой составляющей смеси); 
○ количество компонентов в смеси практически не ограничено, так как аппарат для формирования смеси может быть адаптирован на необходимое количество компонентов без изменения принципиальных основ технологии;

○ в случае, если в качестве одного или нескольких компонентов применяется газ или смесь газов, принципиальные основы технологии позволяют получить аэрозоль в виде смеси или позволяют смешать смесь с сжатым газом или воздухом;

○ технология позволяет вести последовательный ввод дополнительных компонентов в смесь, причём количество таких компонентов и их химический состав не лимитированы;

○ поскольку все параметры смеси зависят от давления в потоке компонентов смеси, регулирование свойств и параметров этой смеси может осуществляться при помощи регулировки давления.

\section{WHAT IS CLAIMED IS...}

1. Первый независимый пункт формулы имеет самое важное значение для заявки:

Первый пункт формулы изобретения должен быть предельно кратким и при этом состоять из трёх частей:

○ В первой части даётся коммерческое название технического решения, которое должно охватывать как можно большую область коммерческого использования, и при этом давать представление о пределах распространения притязаний заявляемого технического решения, исключая рекламные обороты речи и парадоксальные утверждения;

○ Во второй части первого пункта даётся описание отличительных признаков, их совокупностей и взаимосвязей, которые известны и используются как базовые для реализации отличительных признаков;

○ В третьей части первого пункта даётся описание отличительных признаков, их совокупностей и статических и динамических связей отличительных признаков с базовыми известными признаками, которые в совокупности обеспечивают достижение идеального конечного результата.

Последующие зависимые пункты формулы должны более детально характеризовать отличительные признаки в как можно более общей форме. Все последующие пункты формулы должны раскрывать все возможные варианты применения отличительных признаков для формирования эффекта и достижения идеального конечного результата. Если идеальный конечный результат может быть достигнут в некоторых 
вариациях и при условии наличия различных вариантов сочетания отличительных признаков с базовыми признаками, в последующих пунктах указываются все возможные варианты.

Каждый последующий пункт формулы также состоит из трёх частей, требования к каждой из которых соответствует требованиям к первому пункту формулы.

2. Второй независимый пункт формулы изобретения.

3. Зависимый пункт от первого и третьего пунктов формулы изобретения.

4. Зависимый пункт от первого и третьего пунктов формулы изобретения.

5. Зависимый от первого и третьего пунктов формулы изобретения.

6. Зависимый от первого и третьего пунктов формулы изобретения.

7. Третий независимый пункт формулы изобретения:

Если объект изобретения, - устройство или аппарат и названой, - например аппарат, то желательно иметь как минимум три независимых пункта формулы, чтобы первый пункт был, - аппарат для..., метод применения и ассоциированный способ изготовления.

Третий пункт, - метод применения аппарата.

8. Восьмой пункт, - аппарат для... и ассоциированный способ его изготовления.

Всё указанное только пример, каждое техническое решение имеет свои оригинальные особенности и от этого могут меняться состав и структура построения пунктов формулы изобретения.

\section{ABSTRACT OF THE DISCLOSURE}

Аннотация в принципе не отличается чем-то особенным и рекомендаций к оформлению не требует.

\section{СПИСОК ИСПОЛЬЗОВАННОЙ ЛИТЕРАТУРЫ, ПАТЕНТНЫХ И ЛИЦЕНЗИОННЫХ МАТЕРИАЛОВ}

ПРИЛОЖЕНИЕ 1 


\title{
ARTIFICIAL INTELLIGENCE DATA PROCESSING SYSTEM AND METHOD
}

\begin{abstract}
There are provided a system that is capable of performing tasks associated with IPR procurement. The system employs a computing architecture that provides characteristics of artificial intelligence (AI). The computing architecture employs a configuration of pseudo-analog variable-state machines that is implemented by disposing the pseudo-analog variable-state machines in a hierarchical arrangement, wherein pseudo-analog variablestate machines higher in the hierarchical arrangement mimic behavior of a human claustrum for performing higher cognitive functions when processing information associated with one or more service requests and for performing quality checking of the one or more work products. Moreover, the computing architecture is susceptible to being implemented by employing a novel configuration of data processing devices, for example arrays of RISC processors.
\end{abstract}

\section{ПРИЛОЖЕНИЕ 2}

United States Patent Application

20190205606

Kind Code

A1

Zhou; Shaohua Kevin ; et al.

July 4, 2019

\section{METHOD AND SYSTEM FOR ARTIFICIAL INTELLIGENCE BASED MEDICAL IMAGE SEGMENTATION}

\begin{abstract}
Methods and systems for artificial intelligence based medical image segmentation are disclosed. In a method for autonomous artificial intelligence based medical image
\end{abstract}


segmentation, a medical image of a patient is received. A current segmentation context is automatically determined based on the medical image and at least one segmentation algorithm is automatically selected from a plurality of segmentation algorithms based on the current segmentation context. A target anatomical structure is segmented in the medical image using the selected at least one segmentation algorithm.

\title{
ПРИЛОЖЕНИЕ 3
}

\section{UTILIZING ARTIFICIAL INTELLIGENCE TO DETECT OBJECTS OR PATIENT SAFETY EVENTS IN A PATIENT ROOM}

\begin{abstract}
Methods and systems are provided for detecting objects or patient safety events in a patient room. Artificial intelligence is utilized to enhance safety issue recognition capabilities by the methods and systems. Sensors collect a series of images and depth data in a room of a patient. Data (corresponding to images and depth data of an object or patent safety event) is received from the sensors and compared to stored data to identify the object or patient safety event. The images are communicated to a central video monitoring system and a user may be prompted to confirm if the object requires learning or a patient safety event occurred (or identify the object or patient safety event) or to provide additional parameters or actions. A patient safety learning system analyzes the data and incorporates the user response to enhance safety issue recognition capabilities of the system and reduce false alerts.
\end{abstract}

\section{ПРИЛОЖЕНИЕ 4}




\title{
ARTIFICIAL INTELLIGENCE BASED MONITORING OF SOLID STATE DRIVES AND DUAL IN-LINE MEMORY MODULES
}

\begin{abstract}
In embodiments, a memory controller (MC) includes an output interface, and an execution engine (EE) to identify, based on field test results of a die coupled to the MC, initial test results of the die using an artificial neural network (ANN) trained to identify the die from a set of NVM dies based on initial test results of the set of NVM dies obtained at a time of manufacture of the set of dies. The initial test results include a first useful life prediction and the field test results include a second useful life prediction, and the initial test results are regenerated by the ANN to protect their confidentiality. In embodiments, the MC is further to compare the second useful life prediction with the first useful life prediction, to determine a deviation between the two, and output, via the output interface, the deviation to a user.
\end{abstract}

\section{ПРИЛОЖЕНИЕ 5}

United States Patent Application

20190205885

Kind Code

A1

Lim; Chern Jie ; et al.

July 4, 2019

\section{MACHINE LEARNING ENGINE FOR FRAUD DETECTION FOLLOWING LINK SELECTION}

\begin{abstract}
A machine learning engine for fraud detection following link selection may be trained using artificial intelligence techniques and used according to techniques discussed herein. A buyer account may be used to establish and generate a digital gift card having a particular value specified by the buyer. The digital gift card may then be conveyed to another account, such as an email address. The digital gift card may be provided with an online electronic process for redemption and use of the value, for example, by selecting a link and navigating
\end{abstract}


to the process. When the claimer account attempts to utilize the value of the gift card by navigating to the process or otherwise engaging in the electronic process through a device, a risk and fraud analysis engine may execute to determine, based on real-time data of the claimer account, the buyer account, and/or device, whether the digital gift card was generated fraudulently or is being used fraudulently.

\title{
ПРИЛОЖЕНИЕ 6
}

\section{DEVICE OF CONTROLLING RELATED DEVICE USING ARTIFICIAL INTELLIGENCE BASED ON OPERATION SITUATION, SCHEDULE BOT AND SERVER CONTROLLING THEREOF}

\begin{abstract}
This application is a technology with regard to a device that controls a related device based on an operation situation using artificial intelligence, a schedule bot and a server that controls the same, and the device that receives a control of the related device based on the operation situation by an exemplary embodiment of this application includes a function unit that performs a physical function of the device, a communication unit that receives a time schedule and function information from a related device or a schedule management device, and control unit that controls the function unit to maintain a ready state so that a function unit performs a first function at a point that is indicated in the time schedule by using the time schedule and the function information that the communication unit receives.
\end{abstract}

\section{ПРИЛОЖЕНИЕ 7}

United States Patent Application

20190197357

Kind Code 


\title{
MISUSE INDEX FOR EXPLAINABLE ARTIFICIAL INTELLIGENCE IN COMPUTING ENVIRONMENTS
}

\begin{abstract}
A mechanism is described for facilitating misuse index for explainable artificial intelligence in computing environments, according to one embodiment. A method of embodiments, as described herein, includes mapping training data with inference uses in a machine learning environment, where the training data is used for training a machine learning model. The method may further include detecting, based on one or more policy/parameter thresholds, one or more discrepancies between the training data and the inference uses, classifying the one or more discrepancies as one or more misuses, and creating a misuse index listing the one or more misuses.
\end{abstract}

\section{ПРИЛОЖЕНИЕ 8}

United States Patent Application

20190187775

Kind Code

A1

Rotem; Nadav ; et al.

June 20, 2019

\section{DYNAMIC POWER MANAGEMENT FOR ARTIFICIAL INTELLIGENCE HARDWARE ACCELERATORS}

\begin{abstract}
A computer-implemented method for dynamically managing the power usage and/or performance of an artificial intelligence (AI) hardware accelerator may include (1) receiving an instruction stream that includes one or more instructions for performing at least one AI-specific computing task, (2) identifying a plurality of special-purpose, hardware-based functional units configured to perform AI-specific computing tasks, (3) predicting, based on an analysis of at least a portion of the instruction stream, a powerusage requirement for at least one of the functional units when executing the instruction
\end{abstract}


stream, and then (4) modifying, based on the power-usage requirement, the power supplied to at least one of the functional units. Various other methods and systems are also disclosed.

\title{
ПРИЛОЖЕНИЕ 9
}

United States Patent Application

Kind Code

ALDEA LOPEZ; Sergio
20190188570

A1

June 20, 2019

\section{METHODS AND APPARATUS FOR MODEL PARALLELISM IN ARTIFICIAL NEURAL NETWORKS}

\begin{abstract}
The method according to an embodiment comprises automatically controlling allocation, to memories of available hardware resources, of parameters defining computational operations required to calculate an output of at least one layer of neurons of an artificial neural network. The allocation is controlled on the basis of previously-defined allocation data specifying how the operations required to calculate the output of the one layer of neurons are to be allocated to hardware resources to perform the operations. The allocation data is pre-defined using, at least partly, an automatic computer-implemented process, which may include checking before each iteration of the network which of the hardware resources are available to execute that iteration of the network and, if necessary, re-defining the allocation data for that iteration accordingly
\end{abstract}

\section{ПРИЛОЖЕНИЕ 10}

United States Patent Application

20190192258

Kind Code

A1

KANG; Je Hun ; et al.

June 27, 2019 


\title{
AUTOMATIC STEPWISE TOOTH MOVEMENT SYSTEM AND METHOD USING ARTIFICIAL INTELLIGENCE TECHNOLOGY
}

\begin{abstract}
Disclosed is a stepwise automatic orthodontic system and method using an artificial intelligence technique. The method includes: scanning a dental state of a patient by using an intraoral scanner; allowing a server to determine to which group of grouped data of the database the scanned dental data belong; allowing the server to refer to data of the determined group, move a tooth needing orthodontics gradually, and generate a predictive digital orthodontic dental data set; allowing the server to transmit the orthodonticprocessed digital orthodontic dental data set of a patient to a 3D printer, and allowing the $3 \mathrm{D}$ printer to generate and output a dental orthodontic model; and generating a clear aligner by vacuum-compressing a transparent synthetic resin plate to the generated dental orthodontic model through a vacuum former. In the exemplary embodiment of the present invention, the orthodontic patient is clustered or grouped through an unsupervised learning based on the good orthodontic data excluding personal information of the patient, and the tooth moving plan for orthodontics through repeated reinforcement learning satisfying the orthodontic limit condition suggested by the grouped data and the orthodontics textbook for respective steps.
\end{abstract}

\section{ПРИЛОЖЕНИЕ 11}

United States Patent Application

20190188524

Kind Code

A1

He; Lu ; et al.

June 20, 2019

METHOD AND SYSTEM FOR CLASSIFYING AN OBJECT-OF-INTEREST USING AN ARTIFICIAL NEURAL NETWORK

\begin{abstract}
Methods, systems, and techniques for classifying an object-of-interest using an artificial neural network, such as a convolutional neural network. An artificial neural network receives a sample image including the object-of-interest overlaying a background and a sample background image excluding the object-of-interest and corresponding to the background overlaid by the object-of-interest. The object-of-interest is classified using the
\end{abstract}


artificial neural network. The artificial neural network classifies the object-of-interest using the sample background and sample images. Prior to receiving the sample background and sample images the artificial neural network has been trained to classify the object-ofinterest using training image pairs. Each of at least some of the training image pairs includes a first training image that includes a training object-of-interest overlaying a training background and a training background image excluding the training object-ofinterest and corresponding to the training background.

\title{
ПРИЛОЖЕНИЕ 12
}

United States Patent Application

20190171950

Kind Code

A1

Srivastava; Kumar

June 6, 2019

\section{METHOD AND SYSTEM FOR AUTO LEARNING, ARTIFICIAL INTELLIGENCE (AI) APPLICATIONS DEVELOPMENT, OPERATIONALIZATION AND EXECUTION}

\begin{abstract}
Disclosed is a method and a system a method and a system for auto learning, artificial intelligence (AI) applications development, and execution. Various applications or operations may be associated with training environment-agnostic AI models, automated AI app application performance monitoring, fault, quality and performance remediation through prediction of failures or suboptimal performance, privacy and secure AI training and inference mechanism for data and AI model sharing between untrusted parties, and building auto learning applications that can automatically learn and improve.
\end{abstract}

ПРИЛОЖЕНИЕ 13

United States Patent Application

20190171944

Kind Code 


\title{
INTEGRITY EVALUATION OF UNSTRUCTURED PROCESSES USING ARTIFICIAL INTELLIGENCE (AI) TECHNIQUES
}

\begin{abstract}
A process integrity evaluation system ensures integrity of unstructured processes. The process integrity evaluation system handles structured, semi-structured, and unstructured data at massive and large scale. The system provides scalability, secure storage, indexing, knowledge storage, and visualizations of processes by information retrieval, natural language processing, cloud computing, large scale machine learning, knowledge discovery, and other artificial intelligence techniques. Self-provided data, systematically gathered data, and potentially related data from additional sources are incorporated in the process integrity evaluation system which provides the core capabilities of data integrity checking, entity extraction, entity resolution, entity categorization, entity relationship extraction, processes extraction and reconstruction based on knowledge storage, such as knowledge graphs, inference functions, and evaluation computations. After extracting and reconstructing unstructured processes successfully, machine learning functions compute an integrity assurance score, e.g., a similarity, between extracted documents and the internal records in addition to an evaluation result, which can ensure the integrity of the unstructured processes.
\end{abstract}

ПРИЛОЖЕНИЕ 14

United States Patent Application

20190140913

Kind Code

A1

GUIM BERNAT; Francesc ; et al.

May 9, 2019

\section{TECHNIQUES FOR ARTIFICIAL INTELLIGENCE CAPABILITIES AT A NETWORK SWITCH}

\section{Abstract}


Examples include techniques for artificial intelligence (AI) capabilities at a network switch. These examples include receiving a request to register a neural network for loading to an inference resource located at the network switch and loading the neural network based on information included in the request to support an AI service to be provided by users requesting the $\mathrm{AI}$ service.

\title{
ПРИЛОЖЕНИЕ 15
}

\section{DYNAMICALLY MANAGING ARTIFICIAL NEURAL NETWORKS}

\begin{abstract}
In some embodiments, the disclosed subject matter involves using socket layers with a plurality of artificial neural networks in a machine learning system to create customizable inputs and outputs for a machine learning service. The machine learning service may include a plurality of convolutional neural networks and a plurality of pre-trained fully connected neural networks to find the best fits. In an embodiment, when the customized input or output data is not a good fit with the pre-trained artificial neural networks, a socket layer may automatically request additional convolutional layers or new training of a neural network to dynamically manage the machine learning system to accommodate the customized input or customized output. Other embodiments are described and claimed.
\end{abstract}

\section{ПРИЛОЖЕНИЕ 16}

United States Patent Application

20190163714

Kind Code

A1

MA; Yanjun ; et al.

May 30, 2019 


\title{
SEARCH RESULT AGGREGATION METHOD AND APPARATUS BASED ON ARTIFICIAL INTELLIGENCE AND SEARCH ENGINE
}

\begin{abstract}
The present disclosure provides a search result aggregation method and apparatus based on artificial intelligence and a search engine. The method includes: obtaining a query; generating a plurality of search results according to the query; obtaining a plurality of corresponding demand dimensions according to the query; aggregating the plurality of demand dimensions according to the plurality of search results; obtaining an answer corresponding to each demand dimension, and aggregating the answers corresponding to the plurality of demand dimensions according to the aggregated demand dimensions to generate an aggregation result.
\end{abstract}

ПРИЛОЖЕНИЕ 17

United States Patent Application

Kind Code

Emma; Maria ; et al.
20190156222

A1

May 23, 2019

\section{ARTIFICIAL INTELLIGENCE PLATFORM WITH IMPROVED CONVERSATIONAL ABILITY AND PERSONALITY DEVELOPMENT}

\begin{abstract}
System, methods, and apparatuses for an artificial intelligence (AI) toy with improved conversational dialogue and personality development. The AI toy determines responses to stimuli based on user profiles and personality profiles that are developed through user interaction and external media inputs. Natural Language Processing (NLP) and other semantic interaction processing is paired with the profiles to develop AI personality and conversational ability.
\end{abstract}

